\title{
Supporting Information: An efficient coupled-perturbed Kohn-Sham implementation of NMR chemical shift computations with local hybrid functionals and gauge-including atomic orbitals
}

\author{
Caspar Jonas Schattenberg, ${ }^{\dagger}$ Kevin Reiter, ${ }^{\ddagger}$ Florian Weigend, ${ }^{\ddagger}$ and Martin Kaupp ${ }^{*} \dagger$ \\ $\dagger$ Technische Universität Berlin, Institut für Chemie, Theoretische Chemie/Quantenchemie, Sekr. C7, Straße des 17. \\ Juni 135, D-10623, Berlin, Germany \\ $\ddagger$ Karlsruher Institut für Technologie (KIT), Institut für Nanotechnologie, Postfach 3640, D-76021 Karlsruhe, \\ Germany \\ E-mail: martin.kaupp@tu-berlin.de
}

\section{S1 Statistical Quantities}

$$
\begin{aligned}
\mathrm{MSE} & =\frac{1}{N} \sum_{i}^{N} \sigma_{i}-\sigma_{\mathrm{i}, \text { ref. }}, \\
\mathrm{MAE} & =\frac{1}{N} \sum_{i}^{N}\left|\sigma_{i}-\sigma_{\mathrm{i}, \text { ref. }}\right|, \\
\mathrm{SD} & =\sqrt{\frac{1}{N} \sum_{i}^{N}\left(\sigma_{i}-\sigma_{\mathrm{i}, \text { ref. }}\right)^{2}-M S E^{2} .}
\end{aligned}
$$

Additionally in the Supporting Information we use the mean absolute relative error (MARE)

$$
\operatorname{MARE}=\left(\frac{1}{N} \sum_{i}^{N} \frac{\left|\sigma_{i}-\sigma_{\mathrm{i}, \text { ref. }}\right|}{\left|\sigma_{\mathrm{i}, \text { ref. }}\right|}\right) \cdot 100 .
$$

\section{S2 Semi-Numerical Integration}

In the semi-numerical scheme, the four-center integrals are approximated by

$$
\langle\mu \lambda \mid \kappa \nu\rangle \approx \sum_{g} w_{g} \cdot \phi_{\mu, g} \phi_{\lambda, g} \mathrm{~A}_{\kappa \nu, g}
$$

where $w_{g}$ is the weight at each grid point replacing the integration variable $\mathrm{d} \mathbf{r}$. The integration of the A-matrix elements $\mathrm{A}_{\kappa \nu, g}$ is still performed analytically, as mentioned also in the main text. Conveniently, the integration 
can be separated into grid-batches of up to 100 grid points per batch, allowing for efficient parallelization and for a reduction of the memory requirements of the code. In general, the exchange contribution may be expressed as

$$
K_{\mu \nu}=-\frac{1}{2} \sum_{\kappa \lambda} \sum_{g} w_{g} D_{\kappa \lambda} \cdot \phi_{\mu, g} \phi_{\lambda, g} \mathrm{~A}_{\kappa \nu, g}
$$

The term is in practice factorized into pre-calculated contractions of the density matrix with one basis-function vector,

$$
F_{\kappa, g}=\sum_{\lambda} \sqrt{w_{g}} \cdot D_{\kappa \lambda} \phi_{\lambda, g}
$$

in the following multiplied with the (symmetric unperturbed) A-matrix elements $\left(\mathrm{A}_{\nu \kappa, g}=\mathrm{A}_{\kappa \nu, g}\right)$,

$$
G_{\nu, g}=\sum_{\kappa} \mathrm{A}_{\nu \kappa, g} F_{\kappa, g}
$$

In this way, the required storage is minimized even further, as instead of the $N_{\text {bas }}$ A-matrix elements we simply have one $G_{\nu, g}$. Finally, the $G_{\nu, g}$ matrix is contracted with the second basis function and grid weight to give the final exchange contribution,

$$
K_{\mu \nu}=-\frac{1}{2} \sum_{g} \sqrt{w_{g}} \cdot \phi_{\mu, g} G_{\nu, g}
$$

\section{S3 The Perturbed A-Matrix}

Given the perturbed A-matrix (eq. 20 of main text),

$$
\begin{aligned}
\mathrm{A}_{\mu \nu, g}^{B_{k}} & =\left.\int \frac{\left[\omega_{\mu}^{*}(\mathbf{r}) \omega_{\nu}(\mathbf{r})\right]^{B_{k}}}{\left|\mathbf{r}_{g}-\mathbf{r}\right|} \mathrm{d} \mathbf{r}\right|_{B_{k}=0} \\
& =\frac{i}{2 c} \int\left(\mathbf{R}_{\mu \nu} \times \mathbf{r}\right)_{k} \frac{\left[\phi_{\mu}(\mathbf{r}) \phi_{\nu}(\mathbf{r})\right]}{\left|\mathbf{r}_{g}-\mathbf{r}\right|} \mathrm{d} \mathbf{r}
\end{aligned}
$$

we consider as example only one of the Cartesian components $k=x, y, z$, say the $\mathrm{x}$-component selected in the main text. We then have

$$
A_{\mu \nu, g}^{B_{x}}=\frac{i}{2 c}\left\{R_{\mu \nu, y} \int z\left(\phi_{\mu} \phi_{\nu}\right) \frac{1}{\left|\mathbf{r}_{g}-\mathbf{r}\right|} \mathrm{d} \mathbf{r}-R_{\mu \nu, z} \int y\left(\phi_{\mu} \phi_{\nu}\right) \frac{1}{\left|\mathbf{r}_{g}-\mathbf{r}\right|} \mathrm{d} \mathbf{r}\right\} .
$$

Following ref. 1,

- we use the Gaussian expansion scheme $\phi_{\mu}=G_{i j k}\left(\mathbf{r}, a, \mathbf{R}_{\mu}\right)=x_{\mathbf{R}_{\mu}}^{i} y_{\mathbf{R}_{\mu}}^{j} z_{\mathbf{R}_{\mu}}^{k} \exp \left(-a \mathbf{r}_{\mathbf{R}_{\mu}}^{2}\right)$, where $\mathbf{r}_{\mathbf{R}_{\mu}}=\mathbf{r}-\mathbf{R}_{\mu}$ is the position of the basis function.

- The Gaussian function is separable into Cartesian components $G_{i j k}\left(\mathbf{r}, a, \mathbf{R}_{\mu}\right)=G_{i}\left(x, a, R_{\mu, x}\right) G_{j}\left(y, a, R_{\mu, y}\right) G_{k}\left(z, a, R_{\mu, z}\right)$, with $G_{i}\left(x, a, R_{\mu, x}\right)=x_{R_{\mu}}^{i} \exp \left(-a x_{R_{\mu}}^{2}\right)$ 
- The Cartesian resolved overlap is, $\Omega_{i j}^{x}\left(x, a, b, R_{\mu, x}, R_{\nu, x}\right)=G_{i}\left(x, a, R_{\mu, x}\right) G_{j}\left(x, b, R_{\nu, x}\right)$.

Thus, we expand as

$$
\begin{aligned}
A_{\mu \nu, g}^{B_{x}} & =\frac{i}{2 c}\left\{R_{\mu \nu, y} \int z-R_{\mu, z}+R_{\mu, z} \frac{\phi_{\kappa}^{*} \phi_{\nu}}{\left|\mathbf{r}_{g}-\mathbf{r}\right|} \mathrm{d} \mathbf{r}-R_{\mu \nu, z} \int y-R_{\mu, y}+R_{\mu, y} \frac{\phi_{\mu} \phi_{\nu}}{\left|\mathbf{r}_{g}-\mathbf{r}\right|} \mathrm{d} \mathbf{r}\right\} \\
& =\frac{i}{2 c}\left\{R_{\mu \nu, y} \int\left(z_{R_{\mu}}+R_{\mu, z}\right) \frac{\Omega_{i j}^{x} \Omega_{k l}^{y} \Omega_{m n}^{z}}{\left|\mathbf{r}_{g}-\mathbf{r}\right|} \mathrm{d} \mathbf{r}-R_{\mu \nu, z} \int\left(y_{R_{\mu}}+R_{\mu, y}\right) \frac{\Omega_{i j}^{x} \Omega_{k l}^{y} \Omega_{m n}^{z}}{\left|\mathbf{r}_{g}-\mathbf{r}\right|} \mathrm{d} \mathbf{r}\right\} \\
& =\frac{i}{2 c}\left\{R_{\mu \nu, y}\left(A_{\mu \nu}^{m+1}+R_{\mu, z} A_{\mu \nu}\right)-R_{\mu \nu, z}\left(A_{\mu \nu}^{k+1}+R_{\mu, y} A_{\mu \nu}\right)\right\},
\end{aligned}
$$

which gives the perturbed A-matrix of the main text. 
Table S1: Top: Comparison of selected isotropic shielding constants obtained with analytical vs. semi-numerical EXX integration (B3-LYP/pcSseg-4). Bottom: Mean absolute errors (MAE) of the semi-numerical results compared to the analytical program code (in ppm, results for pcSseg-4 and grid setting 5) for various functionals.

\begin{tabular}{|c|c|c|c|c|}
\hline B3-L & $\begin{array}{l}\mathrm{P} \\
\text { molecule }\end{array}$ & analyt. & sem.-num. (eq.27) & sem.-num. $\mathrm{A}^{\mathrm{B}}$ (eq.26) \\
\hline \multirow[t]{8}{*}{${ }^{1} \mathrm{H}$} & furan (C2/5) & 23.998 & 23.998 & 23.998 \\
\hline & furan (C3/4) & 24.995 & 24.995 & 24.995 \\
\hline & $\left(\mathrm{CH}_{3}\right)_{2} \mathrm{CO}$ & 29.800 & 29.800 & 29.800 \\
\hline & $\mathrm{CH}_{4}$ & 31.592 & 31.592 & 31.592 \\
\hline & $\mathrm{NH}_{3}$ & 31.720 & 31.720 & 31.720 \\
\hline & $\mathrm{H}_{2} \mathrm{O}$ & 31.042 & 31.042 & 31.042 \\
\hline & $\mathrm{HF}$ & 29.334 & 29.334 & 29.334 \\
\hline & $\mathrm{PH}_{3}$ & 29.631 & 29.631 & 29.631 \\
\hline \multirow[t]{7}{*}{${ }^{13} \mathrm{C}$} & furan (C2/5) & 26.504 & 26.504 & 26.504 \\
\hline & furan (C3/4) & 61.627 & 61.627 & 61.627 \\
\hline & $\left(\mathrm{CH}_{3}\right)_{2} \underline{\mathrm{CO}}$ & -39.810 & -39.810 & -39.810 \\
\hline & $\left(\mathrm{CH}_{3}\right)_{2} \overline{\mathrm{CO}}$ & 148.840 & 148.840 & 148.840 \\
\hline & $\mathrm{CH}_{4}$ & 188.822 & 188.822 & 188.822 \\
\hline & $\mathrm{CF}_{4}$ & 44.642 & 44.642 & 44.642 \\
\hline & $\mathrm{CO}$ & -22.925 & -22.925 & -22.925 \\
\hline \multirow[t]{5}{*}{${ }^{15} \mathrm{~N}$} & $\mathrm{~N}_{2}$ & -97.205 & -97.205 & -97.205 \\
\hline & $\mathrm{NH}_{3}$ & 259.679 & 259.679 & 259.679 \\
\hline & NNO & -14.516 & -14.516 & -14.516 \\
\hline & $\underline{\mathrm{NNO}}$ & 79.013 & 79.013 & 79.013 \\
\hline & $\mathrm{PN}$ & -447.495 & -447.495 & -447.495 \\
\hline \multirow[t]{6}{*}{${ }^{17} \mathrm{O}$} & furan & 15.031 & 15.031 & 15.031 \\
\hline & $\left(\mathrm{CH}_{3}\right)_{2} \mathrm{CO}$ & -356.208 & -356.208 & -356.208 \\
\hline & $\mathrm{H}_{2} \mathrm{O}$ & 326.534 & 326.534 & 326.534 \\
\hline & $\mathrm{CO}$ & -88.066 & -88.066 & -88.066 \\
\hline & $\mathrm{OF}_{2}$ & -593.761 & -593.761 & -593.761 \\
\hline & NNO & 171.304 & 171.304 & 171.304 \\
\hline \multirow[t]{5}{*}{${ }^{19} \mathrm{~F}$} & $\mathrm{HF}$ & 411.486 & 411.486 & 411.486 \\
\hline & $\mathrm{F}_{2}$ & -259.918 & -259.918 & -259.918 \\
\hline & $\mathrm{CF}_{4}$ & 241.329 & 241.329 & 241.329 \\
\hline & $\mathrm{OF}_{2}$ & -76.603 & -76.603 & -76.603 \\
\hline & $\mathrm{PF}_{3}$ & 199.155 & 199.155 & 199.155 \\
\hline \multirow[t]{9}{*}{${ }^{31} \mathrm{P}$} & $\mathrm{PH}_{3}$ & 560.987 & 560.988 & 560.987 \\
\hline & $\mathrm{PF}_{3}$ & 168.069 & 168.069 & 168.069 \\
\hline & $\mathrm{PN}$ & -70.298 & -70.298 & -70.298 \\
\hline & & functional & MAE (eq.27) & MAE $\mathrm{A}^{\mathrm{B}}$ (eq.26) \\
\hline & & B3-LYP & 0.00001 & 0.00001 \\
\hline & & BHLYP & 0.00005 & 0.00003 \\
\hline & & TPSSh & 0.00001 & 0.00001 \\
\hline & & PBE0 & 0.00002 & 0.00001 \\
\hline & & rtree-Fock & 0.00004 & 0.00002 \\
\hline
\end{tabular}


Table S2: Different coordinate files of $\mathrm{NH}_{3}$ used to test gauge dependence of the implementation.

\begin{tabular}{lrrr}
\hline centered & 0.000000 & 0.000000 & 0.069289 \\
& 0.807968 & 0.466482 & -0.320910 \\
& 0.000002 & -0.932962 & -0.320910 \\
rotated & -0.807969 & 0.466479 & -0.320910 \\
& 0.069289 & 0.000000 & 0.000000 \\
& -0.320910 & 0.807968 & 0.466482 \\
& -0.320910 & 0.000002 & -0.932962 \\
translated & -0.320910 & -0.807969 & 0.466479 \\
& 100.000000 & 100.000000 & 100.069289 \\
& 100.807968 & 100.466482 & 99.679090 \\
& 100.000002 & 99.067038 & 99.679090 \\
& 99.192031 & 100.466479 & 99.679090 \\
\hline
\end{tabular}

Table S3: Isotropic shielding constants for $\mathrm{NH}_{3}$ (in ppm, results for def2-TZVP and pcSseg-4 and grid setting 5) for various functionals using structures indicated in Table S2.

\begin{tabular}{ll|rrr|rrr}
\hline & \multicolumn{3}{|c|}{ def2-TZVP } & \multicolumn{3}{c}{ pcSseg-4 } \\
& functional & cent. & rot. & transl. & cent. & rot. & transl. \\
\hline${ }^{1} \mathrm{H}$ & Hartree-Fock & 32.236 & 32.236 & 32.236 & 31.570 & 31.570 & 31.570 \\
& 32.600 & 32.600 & 32.600 & 31.833 & 31.833 & 31.833 \\
& BLYP & 32.616 & 32.616 & 32.616 & 31.945 & 31.945 & 31.945 \\
TPSS & 32.548 & 32.548 & 32.548 & 31.883 & 31.883 & 31.882 \\
TPSSh & 32.456 & 32.456 & 32.456 & 31.720 & 31.720 & 31.719 \\
B3LYP & 32.307 & 32.307 & 32.307 & 31.594 & 31.594 & 31.594 \\
BHLYP & 32.316 & 32.316 & 32.316 & 31.575 & 31.575 & 31.574 \\
Lh07s-SVWN & 32.076 & 32.076 & 32.076 & 31.326 & 31.326 & 31.326 \\
Lh07t-SVWN & 31.951 & 31.951 & 31.951 & 31.175 & 31.175 & 31.175 \\
Lh12ct-SsirPW92 & 31.882 & 31.882 & 31.882 & 31.094 & 31.094 & 31.094 \\
Lh12ct-SsifPW92 & & & & & & \\
& & 266.193 & 266.193 & 266.187 & 262.073 & 262.073 & 262.067 \\
Hartree-Fock & 265.682 & 265.682 & 265.684 & 258.450 & 258.450 & 258.453 \\
BLYP & 265.878 & 265.878 & 265.881 & 259.258 & 259.258 & 259.261 \\
TPSS & 266.260 & 266.260 & 266.261 & 259.988 & 259.988 & 259.980 \\
TPSSh & 266.122 & 266.122 & 266.115 & 259.679 & 259.679 & 259.660 \\
B3LYP & 266.593 & 266.593 & 266.566 & 261.062 & 261.062 & 260.975 \\
BHLYP & 270.172 & 270.172 & 270.167 & 263.877 & 263.877 & 263.833 \\
Lh07s-SVWN & 280.812 & 280.812 & 280.806 & 275.845 & 275.845 & 275.838 \\
Lh07t-SVWN & 285.085 & 285.085 & 285.082 & 280.429 & 280.429 & 280.417 \\
Lh12ct-SsirPW92 & 286.944 & 286.944 & 286.937 & 282.390 & 282.390 & 282.376 \\
Lh12ct-SsifPW92 & 286.949
\end{tabular}


Table S4: Number of primitive functions $\left(N_{\text {prim }}\right)$ in different basis sets used in timing calculations for ethylene.

\begin{tabular}{lr}
\hline basis set & $N_{\text {prim }}$ \\
\hline def2-SVP & 76 \\
def2-TZVP & 124 \\
def2-TZVPD & 148 \\
def2-QZVP & 286 \\
def2-QZVPD & 310 \\
cc-pVDZ & 96 \\
cc-pVTZ & 164 \\
cc-pVQZ & 282 \\
cc-pV5Z & 468 \\
aug-cc-pVDZ & 130 \\
aug-cc-pVTZ & 232 \\
aug-cc-pVQZ & 396 \\
aug-cc-pV5Z & 640 \\
d-aug-cc-pVDZ & 164 \\
d-aug-cc-pVTZ & 300 \\
d-aug-cc-pVQZ & 510 \\
d-aug-cc-pV5Z & 812 \\
\hline
\end{tabular}



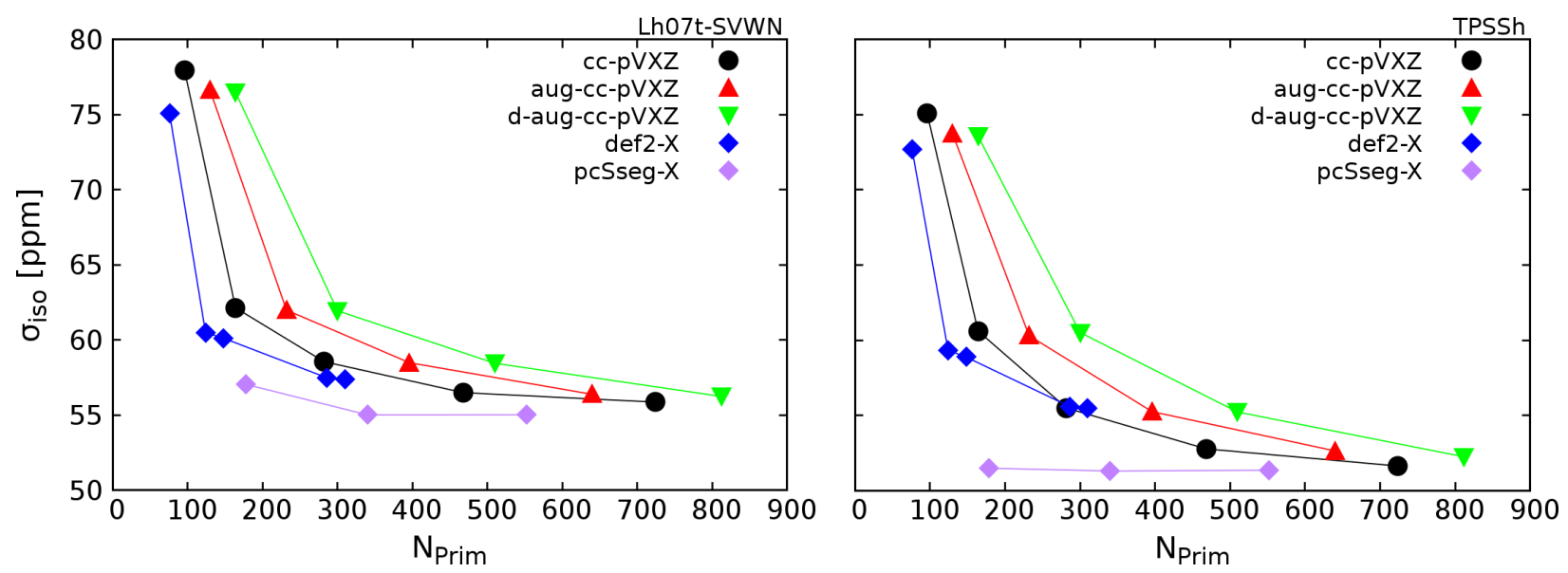

Figure S1: Convergence of isotropic shielding constants (in ppm) for ethylene with LH07t-SVWN (left) and TPSSh (right) functionals, using various basis sets from the def2-X (X = SVP, TZVP, TZVPD, QZVP, QZVPD) and ((d)aug-)cc-pVXZ (X= D, T, Q, 5) families, as well as cc-pV6Z and pcSseg-X $(\mathrm{X}=2,3,4)$
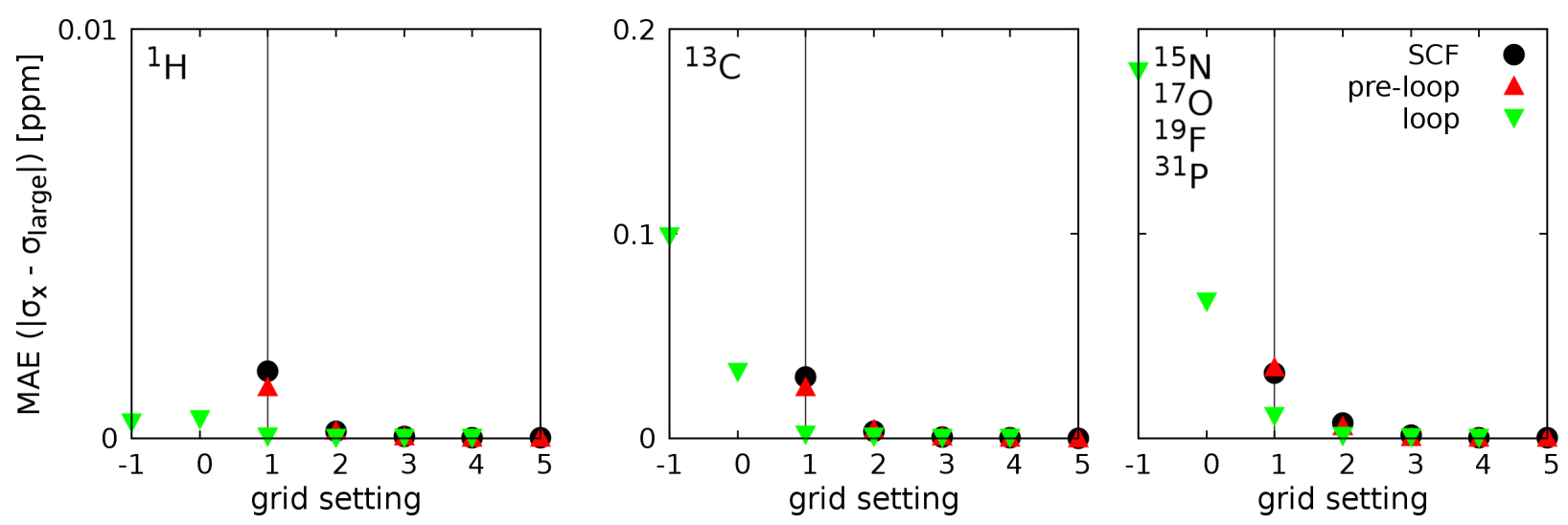

Figure S2: MAEs of shieldings obtained with various grid sizes relative to the extra-large $(7 / 25)$ grid at lh07tsvwn/pcSseg-4/MARI-J level for ${ }^{1} \mathrm{H}$ (left), ${ }^{13} \mathrm{C}$ (middle), and the other nuclei $\left({ }^{15} \mathrm{~N},{ }^{17} \mathrm{O},{ }^{19} \mathrm{~F}\right.$ and ${ }^{31} \mathrm{P}$, right). See Tables S5-S7 for full numerical data. 
Table S5: Absolute $\Delta \sigma_{a b s}$ (in ppm) and relative deviations $\Delta \sigma_{r e l}$ (in \%) due to grid settings (g1-g5, according to Table 1 in main text) in ground-state SCF at Lh07t-SVWN/pcSseg-4/MARI-J level

\begin{tabular}{|c|c|c|c|c|c|c|c|c|c|c|c|c|}
\hline \multicolumn{3}{|c|}{ SCF grid scan } & \multicolumn{5}{|c|}{$\Delta \sigma_{a b s}[\mathrm{ppm}]$} & \multicolumn{5}{|c|}{$\Delta \sigma_{\text {rel }}[\%]$} \\
\hline & molecule & $\operatorname{grid} 7 / 25$ & g1 & g2 & g3 & g4 & g5 & g1 & g2 & g3 & $\mathrm{g} 4$ & g5 \\
\hline \multirow[t]{8}{*}{${ }^{1} \mathrm{H}$} & furan $(\mathrm{C} 2 / 5)$ & 23.681 & 0.002 & 0.000 & 0.000 & 0.000 & 0.000 & 0.008 & 0.001 & 0.000 & 0.000 & 0.000 \\
\hline & furan $(\mathrm{C} 3 / 4)$ & 24.626 & 0.003 & 0.000 & 0.000 & 0.000 & 0.000 & 0.012 & 0.000 & 0.001 & 0.000 & 0.000 \\
\hline & $\left(\mathrm{CH}_{3}\right)_{2} \mathrm{CO}$ & 29.283 & .002 & 0.000 & 0.000 & 0.000 & 0.000 & 0.007 & 0.002 & 0.000 & 0.000 & 0.000 \\
\hline & $\mathrm{CH}_{4}$ & 31.307 & 0.002 & 0.000 & 0.000 & 0.000 & 0.000 & 0.006 & 0.000 & 0.000 & 0.000 & 0.000 \\
\hline & $\mathrm{NH}_{3}$ & 1.326 & 0.001 & 0.000 & 0.000 & 0.000 & 0.000 & 0.004 & 0.001 & 0.000 & 0.000 & 0.000 \\
\hline & $\mathrm{H}_{2} \mathrm{O}$ & 0.474 & 001 & 0.000 & 0.000 & 0.000 & .000 & 0.004 & 0.000 & 0.000 & 0.000 & 0.000 \\
\hline & $\mathrm{HF}$ & 28.552 & 0.001 & 0.000 & 0.000 & 0.000 & 0.000 & 0.004 & 0.000 & 0.000 & 0.000 & 0.000 \\
\hline & $\mathrm{PH}_{3}$ & 29.251 & 0.000 & 0.000 & 0.000 & 0.000 & 0.000 & 0.002 & 0.000 & 0.000 & 0.000 & 0.000 \\
\hline \multirow[t]{7}{*}{${ }^{13} \mathrm{C}$} & furan $(\mathrm{C} 2 / 5)$ & 38.015 & 0.003 & 0.002 & 0.000 & 0.000 & 0.000 & 0.007 & 0.006 & 0.000 & 0.000 & 0.000 \\
\hline & furan $(\mathrm{C} 3 / 4)$ & 72.317 & 0.029 & 0.006 & 0.002 & 0.000 & 0.000 & 0.040 & 0.008 & 0.002 & 0.000 & 0.000 \\
\hline & $\left(\mathrm{CH}_{3}\right)_{2} \underline{\mathrm{CO}}$ & -30.312 & 0.038 & 0.001 & 0.000 & 0.000 & 0.000 & 0.124 & 0.004 & 0.001 & 0.000 & 0.001 \\
\hline & $\left(\mathrm{CH}_{3}\right)_{2} \mathrm{CO}$ & 9.382 & 0.093 & 0.010 & 0.001 & 0.000 & 0.000 & .058 & 0.006 & 0.001 & 00 & .000 \\
\hline & $\overline{\mathrm{CH}}_{4}$ & 9.852 & 0.031 & 0.002 & 0.000 & 0.000 & 0.000 & 0.015 & 0.001 & 0.000 & 0.000 & 0.000 \\
\hline & $\mathrm{CF}_{4}$ & 3.404 & 0.013 & 0.001 & 0.000 & 0.000 & 0.000 & 0.025 & 0.003 & 0.001 & 0.000 & 0.000 \\
\hline & $\mathrm{CO}$ & 202 & 0.002 & 0.001 & 0.000 & 0.000 & 0.000 & 0.018 & 0.007 & 0.002 & 0.001 & 0.001 \\
\hline \multirow[t]{5}{*}{${ }^{15} \mathrm{~N}$} & $\mathrm{~N}_{2}$ & -81.592 & 0.001 & 0.000 & 0.000 & 0.000 & 0.000 & 0.001 & 0.000 & 0.000 & 0.001 & 0.000 \\
\hline & $\mathrm{NH}_{3}$ & 275.845 & 034 & 0.000 & 0.000 & 0.000 & 0.000 & 0.012 & 0.000 & 0.000 & 0.000 & 0.000 \\
\hline & NNO & -1.517 & .002 & 0.000 & 0.000 & 0.000 & 000 & 132 & 013 & 0.010 & 0.007 & 0.009 \\
\hline & $\underline{\mathrm{NNO}}$ & 94.444 & 0.002 & 0.000 & 0.000 & .000 & 000 & 0.002 & 0.000 & 0.000 & 0.000 & 0.000 \\
\hline & $\overline{\mathrm{PN}}$ & -401.714 & 0.020 & 0.001 & 0.000 & 0.000 & 0.000 & 0.005 & 0.000 & 0.000 & 0.000 & 0.000 \\
\hline \multirow[t]{6}{*}{${ }^{17} \mathrm{O}$} & furan & & 0.091 & 0.016 & 0.004 & 0.000 & & 0.242 & 0.042 & 0.012 & 0.001 & 0.000 \\
\hline & $\left(\mathrm{CH}_{3}\right)_{2} \mathrm{CO}$ & -316.519 & .066 & 0.060 & 0.007 & .000 & 0.001 & 0.021 & 0.019 & 0.002 & 0.000 & 0.000 \\
\hline & $\mathrm{H}_{2} \mathrm{O}$ & 19.495 & .037 & 0.001 & 0.000 & .000 & 0.000 & 0.011 & 0.000 & 0.000 & 0.000 & 0.000 \\
\hline & $\mathrm{CO}$ & 4.958 & .009 & 0.003 & 0.001 & 000 & 000 & 0.014 & 0.005 & 0.001 & 0.000 & 0.000 \\
\hline & $\mathrm{OF}_{2}$ & 38.323 & 0.027 & 0.011 & 0.006 & 0.000 & 001 & 0.005 & 0.002 & 0.001 & 0.000 & 0.000 \\
\hline & $\mathrm{NNO}$ & & 0.005 & 0.000 & 0.000 & 0.000 & 0.000 & 0.003 & 0.000 & 0.000 & 0.000 & 0.000 \\
\hline \multirow[t]{5}{*}{${ }^{19} \mathrm{~F}$} & $\mathrm{HF}$ & & 0.001 & & & & & 0.000 & & & & 0.000 \\
\hline & $\mathrm{F}_{2}$ & -215.668 & 016 & 0.000 & 0.003 & .000 & 0.000 & .007 & 0.000 & 0.001 & 0.000 & 0.000 \\
\hline & $\mathrm{CF}_{4}$ & 262.882 & .010 & 0.000 & 0.001 & 0.000 & 0.000 & 0.004 & 0.000 & 0.000 & 0.000 & 0.000 \\
\hline & $\mathrm{OF}_{2}$ & -34.200 & 0.020 & 0.003 & 0.001 & 0.000 & & 0.059 & 0.009 & 0.002 & 0.001 & 0.001 \\
\hline & $\mathrm{PF}_{3}$ & 227.022 & 0.031 & 0.004 & 0.000 & 0.000 & 0.000 & 0.014 & 0.002 & 0.000 & 0.000 & 0.000 \\
\hline \multirow[t]{3}{*}{${ }^{31} \mathrm{P}$} & $\mathrm{PH}_{3}$ & & .145 & 0.025 & 0.000 & 0.000 & 0.000 & 0.024 & 0.004 & 0.000 & 0.000 & 0.000 \\
\hline & $\mathrm{PF}_{3}$ & & 0.035 & 0.009 & 0.001 & 0.000 & 0.000 & 0.019 & 0.005 & 0.001 & 0.000 & 0.000 \\
\hline & $\mathrm{PN}$ & -44.887 & 0.052 & 0.005 & 0.001 & 0.001 & 0.001 & 0.115 & 0.011 & 0.002 & 0.003 & 0.00 \\
\hline \multicolumn{3}{|c|}{ MAE/MARE $\left({ }^{1} \mathrm{H}\right)$} & 0.002 & 0.000 & 0.000 & 0.000 & 0.000 & 0.006 & 0.001 & 0.000 & 0.000 & 0.000 \\
\hline \multicolumn{3}{|c|}{ MAE/MARE $\left({ }^{13} \mathrm{C}\right)$} & 0.030 & 0.003 & 0.001 & 0.000 & & 0.041 & 0.005 & 0.001 & 0.000 & 0.000 \\
\hline \multicolumn{3}{|c|}{ MAE/MARE (other) } & 0.032 & 0.007 & 0.001 & 0.000 & 0.000 & 0.036 & 0.006 & 0.002 & 0.001 & 0.001 \\
\hline
\end{tabular}


Table S6: Absolute $\Delta \sigma_{a b s}$ (in ppm) and relative deviations $\Delta \sigma_{r e l}$ (in \%) due to grid setting (g1-g5, according to Table 1 in main text) in the pre-loop part of the shielding calculation at Lh07t-SVWN/pcSseg-4/MARI-J level

\begin{tabular}{|c|c|c|c|c|c|c|c|c|c|c|c|c|}
\hline \multicolumn{3}{|c|}{ pre-loop grid scan } & \multicolumn{5}{|c|}{$\Delta \sigma_{a b s}($ in $\mathrm{ppm})$} & \multicolumn{5}{|c|}{$\Delta \sigma_{r e l}($ in $\%)$} \\
\hline & molecule & $\operatorname{grid} 7 / 25$ & g1 & g2 & g3 & g4 & g5 & g1 & g2 & g3 & $\mathrm{g} 4$ & $g$ \\
\hline \multirow[t]{8}{*}{$\overline{{ }^{1} \mathrm{H}}$} & furan $(\mathrm{C} 2 / 5)$ & 23.681 & 0.001 & 0.000 & 0.000 & 0.000 & 0.000 & 0.004 & 0.002 & 0.000 & 0.000 & 0.000 \\
\hline & furan $(\mathrm{C} 3 / 4)$ & & .004 & 0.000 & 0.000 & 0.000 & 0.000 & 0.017 & 0.001 & 0.001 & 0.000 & 0.000 \\
\hline & $\left(\mathrm{CH}_{3}\right)_{2} \mathrm{CO}$ & 29.283 & .001 & 0.000 & 0.000 & 0.000 & 0.000 & 0.003 & 0.002 & 0.000 & 0.000 & 0.000 \\
\hline & $\mathrm{CH}_{4}$ & 1.307 & 0.000 & 0.000 & 0.000 & 0.000 & 0.000 & 0.001 & 0.000 & 0.000 & 0.000 & 0.000 \\
\hline & $\mathrm{NH}_{3}$ & 1.326 & 0.002 & 0.000 & 0.000 & 0.000 & 0.000 & 0.006 & 0.001 & 0.000 & 0.000 & 0.000 \\
\hline & $\mathrm{H}_{2} \mathrm{O}$ & 0.474 & 002 & 0.000 & 0.000 & 0.000 & .000 & 0.005 & 0.000 & 0.000 & 0.000 & 0.000 \\
\hline & $\mathrm{HF}$ & 28.552 & 0.000 & 0.000 & 0.000 & 0.000 & 0.000 & 0.000 & 0.000 & 0.000 & 0.000 & 0.000 \\
\hline & $\mathrm{PH}_{3}$ & 29.251 & 0.000 & 0.000 & 0.000 & 0.000 & 0.000 & 0.000 & 0.000 & 0.000 & 0.000 & 0.000 \\
\hline \multirow[t]{7}{*}{${ }^{13} \mathrm{C}$} & furan $(\mathrm{C} 2 / 5)$ & 38.015 & 0.036 & 0.000 & 0.001 & 0.000 & 0.000 & 0.095 & 0.001 & 0.002 & 0.000 & 0.000 \\
\hline & furan $(\mathrm{C} 3 / 4)$ & & .007 & 0.002 & 0.000 & 0.000 & 0.000 & 0.009 & 0.003 & 0.000 & 0.000 & 0.000 \\
\hline & $\left(\mathrm{CH}_{3}\right)_{2} \underline{\mathrm{CO}}$ & & 0.043 & 0.003 & & 0.000 & & 0.141 & 0.009 & 0.003 & 0.001 & 0.001 \\
\hline & $\left(\mathrm{CH}_{3}\right)_{2} \overline{\mathrm{CO}}$ & 9.382 & 0.061 & 0.011 & 0.001 & 0.000 & 000 & 038 & .007 & 0.001 & 000 & 0.000 \\
\hline & $\overline{\mathrm{CH}}_{4}$ & 9.852 & .023 & 0.012 & 0.001 & 0.000 & 0.000 & 0.011 & 0.006 & 0.001 & 0.000 & 0.000 \\
\hline & $\mathrm{CF}_{4}$ & & & 0.002 & & & & & 0.003 & 0.001 & 0.000 & 0.000 \\
\hline & $\mathrm{CO}$ & 202 & 0.001 & 0.000 & 0.000 & 0.000 & 0.000 & 0.008 & 0.001 & 0.001 & 0.001 & 0.001 \\
\hline \multirow[t]{5}{*}{${ }^{15} \mathrm{~N}$} & $\mathrm{~N}_{2}$ & & 0.001 & 0.000 & 0.000 & 0.000 & 0.000 & 0.001 & 0.001 & 0.000 & 0.000 & 0.000 \\
\hline & $\mathrm{NH}_{3}$ & 275.845 & 032 & 0.005 & 0.000 & 0.000 & 000 & .011 & 0.002 & 0.000 & 0.000 & 0.000 \\
\hline & NNO & -1.517 & 000 & 0.000 & 0.000 & 0.000 & 000 & 08 & 0.006 & 010 & 0.008 & 0.009 \\
\hline & $\underline{\mathrm{NNO}}$ & 94.444 & 0.000 & 0.000 & 0.000 & 0.000 & & 0.000 & 0.000 & 0.000 & 0.000 & 0.000 \\
\hline & $\mathrm{PN}$ & -401.714 & 0.002 & 0.000 & 0.000 & 0.000 & 0.000 & 0.000 & 0.000 & 0.000 & 0.000 & 0.000 \\
\hline \multirow[t]{6}{*}{${ }^{17} \mathrm{O}$} & furan & & 0.044 & 0.013 & & 0.000 & & 0.117 & 0.036 & & & 0.000 \\
\hline & $\left(\mathrm{CH}_{3}\right)_{2} \mathrm{CO}$ & 6.519 & 241 & 0.037 & 0.001 & 0.000 & 001 & 0.076 & 0.012 & 0.000 & 0.000 & 0.000 \\
\hline & $\mathrm{H}_{2} \mathrm{O}$ & 19.495 & .013 & 0.001 & 0.000 & 0.000 & 0.000 & 0.004 & 0.000 & 0.000 & 0.000 & 0.000 \\
\hline & $\mathrm{CO}$ & & 004 & 0.000 & & & & 0.005 & & & 0.000 & 0.00 \\
\hline & $\mathrm{OF}_{2}$ & 38.323 & 0.011 & 0.000 & 0.001 & 0.001 & 001 & 0.002 & 0.000 & 0.000 & 0.000 & 0.000 \\
\hline & $\mathrm{NNO}$ & & 0.000 & 0.000 & 0.000 & 0.000 & 0.000 & 0.000 & 0.000 & 0.000 & 0.000 & 0.000 \\
\hline \multirow[t]{5}{*}{${ }^{19} \mathrm{~F}$} & $\mathrm{HF}$ & & 0.004 & & & & & 0.001 & & & & 0.000 \\
\hline & $\mathrm{F}_{2}$ & 215.668 & .002 & 0.000 & .000 & 0.000 & 0.000 & .001 & 0.000 & 0.000 & 0.000 & 0.000 \\
\hline & $\mathrm{CF}_{4}$ & & 0.044 & 0.004 & 0.000 & & & 0.017 & 0.001 & 0.000 & 0.000 & 0.000 \\
\hline & $\mathrm{OF}_{2}$ & & 0.003 & 0.001 & 0.000 & & & 0.008 & 0.003 & 0.001 & 0.001 & 0.00 \\
\hline & $\mathrm{PF}_{3}$ & 227.022 & 0.051 & 0.008 & 0.001 & 0.000 & 0.000 & 0.022 & 0.003 & 0.000 & 0.000 & 0.000 \\
\hline \multirow[t]{3}{*}{${ }^{31} \mathrm{P}$} & $\mathrm{PH}_{3} \mathrm{t}$ & & 0.204 & 0.039 & 0.002 & 0.001 & 0.000 & 0.034 & 0.007 & 0.000 & 0.000 & 0.000 \\
\hline & $\mathrm{PF}_{3}$ & & 0.000 & 0.000 & 0.000 & 0.000 & 0.000 & 0.000 & 0.000 & 0.000 & 0.000 & 0.000 \\
\hline & $\mathrm{PN}$ & -44.887 & 0.002 & 0.001 & 0.001 & 0.001 & 0.001 & 0.005 & 0.003 & 0.002 & 0.003 & 0.00 \\
\hline \multirow{3}{*}{\multicolumn{3}{|c|}{$\begin{array}{l}\text { MAE/MARE }\left({ }^{1} \mathrm{H}\right) \\
\text { MAE/MARE }\left({ }^{13} \mathrm{C}\right) \\
\text { MAE/MARE (other) }\end{array}$}} & 0.001 & 0.000 & 0.000 & 0.000 & 0.000 & 0.005 & 0.001 & 0.000 & 0.000 & 0.000 \\
\hline & & & 0.025 & 0.004 & 0.001 & 0.000 & & & & 0.001 & 0.000 & 0.000 \\
\hline & & & 0.035 & 0.006 & 0.000 & 0.000 & 0.000 & 0.017 & 0.004 & 0.001 & 0.001 & 0.001 \\
\hline
\end{tabular}


Table S7: Absolute $\Delta \sigma_{a b s}$ (in ppm) and relative deviations $\Delta \sigma_{r e l}$ (in \%) due to grid setting (g-1-g5, according to Table 1 in main text) in CPKS-loop at Lh07t-SVWN/pcSseg-4/MARI-J level

\begin{tabular}{|c|c|c|c|c|c|c|c|c|c|c|c|c|c|c|}
\hline \multicolumn{3}{|c|}{ CPKS-loop grid scan } & \multicolumn{6}{|c|}{$\Delta \sigma_{a b s}($ in $\mathrm{ppm})$} & \multicolumn{6}{|c|}{$\Delta \sigma_{r e l}($ in $\%)$} \\
\hline & molecule & grid $7 / 25$ & $g-1$ & g0 & g1 & g2 & g3 & $\mathrm{g} 4$ & g-1 & g0 & g1 & g2 & g3 & $\mathrm{g} 4$ \\
\hline \multirow[t]{8}{*}{${ }^{1} \mathrm{H}$} & furan $(\mathrm{C} 2 / 5)$ & 23.681 & 0.000 & 0.002 & 0.000 & 0.000 & 0.000 & 0.000 & 0.002 & 0.006 & 0.000 & 0.000 & 0.000 & 0.000 \\
\hline & furan $(\mathrm{C} 3 / 4)$ & 24.626 & 0.001 & 0.001 & 0.000 & 0.000 & 0.000 & 0.000 & 0.005 & 0.003 & 0.001 & 0.000 & 0.000 & 0.000 \\
\hline & $\left(\mathrm{CH}_{3}\right)_{2} \mathrm{CO}$ & 29.283 & 0.001 & 0.001 & 0.000 & 0.000 & 0.000 & 0.000 & 0.002 & 0.002 & 0.000 & 0.000 & 0.000 & 0.000 \\
\hline & $\mathrm{CH}_{4}$ & 31.307 & 0.000 & 0.000 & 0.000 & 0.000 & 0.000 & 0.000 & 0.001 & 0.001 & 0.000 & 0.000 & 0.000 & 0.000 \\
\hline & $\mathrm{NH}_{3}$ & 31.326 & 0.000 & 0.000 & 0.000 & 0.000 & 0.000 & 0.000 & 0.000 & 0.000 & 0.000 & 0.000 & 0.000 & 0.000 \\
\hline & $\mathrm{H}_{2} \mathrm{O}$ & 30.474 & 0.000 & 0.000 & 0.000 & 0.000 & 0.000 & 0.000 & 0.000 & 0.001 & 0.000 & 0.000 & 0.000 & 0.000 \\
\hline & $\mathrm{HF}$ & 28.552 & 0.000 & 0.000 & 0.000 & 0.000 & 0.000 & 0.000 & 0.001 & 0.000 & 0.000 & 0.000 & 0.000 & 0.000 \\
\hline & $\mathrm{PH}_{3}$ & 29.251 & 0.000 & 0.000 & 0.000 & 0.000 & 0.000 & 0.000 & 0.000 & 0.001 & 0.000 & 0.000 & 0.000 & 0.000 \\
\hline \multirow[t]{7}{*}{${ }^{13} \mathrm{C}$} & furan $(\mathrm{C} 2 / 5)$ & 38.015 & 0.116 & 0.026 & 0.000 & 0.001 & 0.000 & 0.000 & 0.305 & 0.069 & 0.001 & 0.003 & 0.000 & 0.000 \\
\hline & furan $(\mathrm{C} 3 / 4)$ & 72.317 & 0.093 & 0.045 & 0.003 & 0.001 & 0.000 & 0.000 & 0.129 & 0.062 & 0.004 & 0.001 & 0.000 & 0.000 \\
\hline & $\left(\mathrm{CH}_{3}\right)_{2} \underline{\mathrm{CO}}$ & -30.312 & 0.194 & 0.053 & 0.008 & 0.002 & 0.000 & 0.000 & 0.640 & 0.174 & 0.027 & 0.006 & 0.001 & 0.001 \\
\hline & $\left(\mathrm{CH}_{3}\right)_{2} \overline{\mathrm{CO}}$ & 159.382 & 0.020 & 0.026 & 0.000 & 0.001 & 0.000 & 0.000 & 0.013 & 0.016 & 0.000 & 0.001 & 0.000 & 0.000 \\
\hline & $\overline{\mathrm{CH}}_{4}$ & 199.852 & 0.011 & 0.009 & 0.000 & 0.000 & 0.000 & 0.000 & 0.006 & 0.004 & 0.000 & 0.000 & 0.000 & 0.000 \\
\hline & $\mathrm{CF}_{4}$ & 53.404 & 0.021 & 0.037 & 0.001 & 0.000 & 0.000 & 0.000 & 0.040 & 0.069 & 0.002 & 0.001 & 0.000 & 0.000 \\
\hline & $\mathrm{CO}$ & -13.202 & 0.234 & 0.032 & 0.000 & 0.001 & 0.000 & 0.000 & 1.774 & 0.239 & 0.002 & 0.010 & 0.002 & 0.001 \\
\hline \multirow[t]{5}{*}{${ }^{15} \mathrm{~N}$} & $\mathrm{~N}_{2}$ & -81.592 & 0.398 & 0.052 & 0.002 & 0.001 & 0.000 & 0.000 & 0.488 & 0.064 & 0.002 & 0.001 & 0.000 & 0.000 \\
\hline & $\mathrm{NH}_{3}$ & 275.845 & 0.029 & 0.014 & 0.002 & 0.001 & 0.000 & 0.000 & 0.010 & 0.005 & 0.001 & 0.000 & 0.000 & 0.000 \\
\hline & NNO & -1.517 & 0.156 & 0.012 & 0.001 & 0.000 & 0.000 & 0.000 & 10.291 & 0.810 & 0.075 & 0.016 & 0.009 & 0.011 \\
\hline & $\underline{\mathrm{NNO}}$ & 94.444 & 0.177 & 0.028 & 0.002 & 0.000 & 0.000 & 0.000 & 0.187 & 0.030 & 0.002 & 0.000 & 0.000 & 0.000 \\
\hline & $\overline{\mathrm{PN}}$ & -401.714 & 0.725 & 0.024 & 0.031 & 0.001 & 0.001 & 0.001 & 0.181 & 0.006 & 0.008 & 0.000 & 0.000 & 0.000 \\
\hline \multirow[t]{6}{*}{${ }^{17} \mathrm{O}$} & furan & 37.547 & 0.136 & 0.033 & 0.006 & 0.003 & 0.000 & 0.000 & 0.363 & 0.087 & 0.016 & 0.007 & 0.001 & 0.000 \\
\hline & $\left(\mathrm{CH}_{3}\right)_{2} \mathrm{CO}$ & -316.519 & 0.024 & 0.325 & 0.029 & 0.001 & 0.000 & 0.001 & 0.008 & 0.103 & 0.009 & 0.000 & 0.000 & 0.000 \\
\hline & $\mathrm{H}_{2} \mathrm{O}$ & 349.495 & 0.001 & 0.006 & 0.005 & 0.000 & 0.000 & 0.000 & 0.000 & 0.002 & 0.001 & 0.000 & 0.000 & 0.000 \\
\hline & $\mathrm{CO}$ & -64.958 & 0.001 & 0.007 & 0.002 & 0.002 & 0.001 & 0.000 & 0.001 & 0.010 & 0.003 & 0.003 & 0.001 & 0.000 \\
\hline & $\mathrm{OF}_{2}$ & -538.323 & 0.843 & 0.075 & 0.030 & 0.003 & 0.000 & 0.001 & 0.157 & 0.014 & 0.005 & 0.001 & 0.000 & 0.000 \\
\hline & $\mathrm{NNO}$ & 191.339 & 0.004 & 0.005 & 0.001 & 0.000 & 0.000 & 0.000 & 0.002 & 0.003 & 0.001 & 0.000 & 0.000 & 0.000 \\
\hline \multirow[t]{5}{*}{${ }^{19} \mathrm{~F}$} & $\mathrm{HF}$ & 437.037 & 0.002 & 0.002 & 0.001 & 0.000 & 0.000 & 0.000 & 0.000 & 0.000 & 0.000 & 0.000 & 0.000 & 0.000 \\
\hline & $\mathrm{F}_{2}$ & -215.668 & 0.356 & 0.183 & 0.004 & 0.001 & 0.000 & 0.000 & 0.165 & 0.085 & 0.002 & 0.000 & 0.000 & 0.000 \\
\hline & $\mathrm{CF}_{4}$ & 262.882 & 0.109 & 0.110 & 0.009 & 0.001 & 0.000 & 0.000 & 0.041 & 0.042 & 0.003 & 0.001 & 0.000 & 0.000 \\
\hline & $\mathrm{OF}_{2}$ & -34.200 & 0.016 & 0.118 & 0.005 & 0.000 & 0.000 & 0.000 & 0.048 & 0.344 & 0.015 & 0.001 & 0.001 & 0.000 \\
\hline & $\mathrm{PF}_{3}$ & 227.022 & 0.022 & 0.069 & 0.021 & 0.004 & 0.000 & 0.000 & 0.010 & 0.030 & 0.009 & 0.002 & 0.000 & 0.000 \\
\hline \multirow[t]{3}{*}{${ }^{31} \mathrm{P}$} & $\mathrm{PH}_{3}$ & 598.967 & 0.020 & 0.044 & 0.004 & 0.001 & 0.001 & 0.000 & 0.003 & 0.007 & 0.001 & 0.000 & 0.000 & 0.000 \\
\hline & $\mathrm{PF}_{3}$ & 189.238 & 0.010 & 0.147 & 0.009 & 0.001 & 0.000 & 0.000 & 0.005 & 0.078 & 0.005 & 0.000 & 0.000 & 0.000 \\
\hline & $\mathrm{PN}$ & -44.887 & 0.380 & 0.015 & 0.046 & 0.001 & 0.003 & 0.001 & 0.848 & 0.034 & 0.103 & 0.002 & 0.006 & 0.002 \\
\hline \multicolumn{3}{|c|}{ MAE/MARE $\left({ }^{1} \mathrm{H}\right)$} & 0.000 & 0.000 & 0.000 & 0.000 & 0.000 & 0.000 & 0.001 & 0.002 & 0.000 & 0.000 & 0.000 & 0.000 \\
\hline \multicolumn{3}{|c|}{ MAE/MARE $\left({ }^{13} \mathrm{C}\right)$} & 0.099 & 0.032 & 0.002 & 0.001 & 0.000 & 0.000 & 0.415 & 0.091 & 0.005 & 0.003 & 0.000 & 0.000 \\
\hline \multicolumn{3}{|c|}{ MAE/MARE (other) } & 0.179 & 0.067 & 0.011 & 0.001 & 0.000 & 0.000 & 0.674 & 0.092 & 0.014 & 0.002 & 0.001 & 0.001 \\
\hline
\end{tabular}


Table S8: Absolute $\Delta \sigma_{a b s}$ (in ppm) and relative deviations $\Delta \sigma_{r e l}$ (in \%) due to combined small-grid settings (grid 1 for SCF, grid 1 for CPKS pre-loop, and grid 0 for CPKS-loop, according to Table 1) at Lh07t-SVWN/pcSseg-4/MARI-J level

\begin{tabular}{|c|c|c|c|c|c|}
\hline \multicolumn{6}{|c|}{ combined small-grid settings } \\
\hline \multirow[t]{8}{*}{${ }^{1} \mathrm{H}$} & furan $(\mathrm{C} 2 / 5)$ & 23.681 & 23.683 & 0.001 & 0.006 \\
\hline & furan $(\mathrm{C} 3 / 4)$ & 24.626 & 24.627 & 0.001 & 0.005 \\
\hline & $\left(\mathrm{CH}_{3}\right)_{2} \mathrm{CO}$ & 29.283 & 29.281 & 0.001 & 0.005 \\
\hline & $\mathrm{CH}_{4}$ & 31.307 & 31.309 & 0.002 & 0.006 \\
\hline & $\mathrm{NH}_{3}$ & 31.326 & 31.326 & 0.000 & 0.001 \\
\hline & $\mathrm{H}_{2} \mathrm{O}$ & 30.474 & 30.474 & 0.000 & 0.001 \\
\hline & $\mathrm{HF}$ & 28.552 & 28.551 & 0.001 & 0.004 \\
\hline & $\mathrm{PH}_{3}$ & 29.251 & 29.250 & 0.001 & 0.002 \\
\hline \multirow[t]{7}{*}{${ }^{13} \mathrm{C}$} & furan $(\mathrm{C} 2 / 5)$ & 38.015 & 38.009 & 0.006 & 0.016 \\
\hline & furan $(\mathrm{C} 3 / 4)$ & 72.317 & 72.405 & 0.088 & 0.121 \\
\hline & $\left(\mathrm{CH}_{3}\right)_{2} \underline{\mathrm{CO}}$ & -30.312 & -30.363 & 0.051 & 0.169 \\
\hline & $\left(\underline{\mathrm{CH}}_{3}\right)_{2} \overline{\mathrm{CO}}$ & 159.382 & 159.429 & 0.047 & 0.030 \\
\hline & $\mathrm{CH}_{4}$ & 199.852 & 199.820 & 0.032 & 0.016 \\
\hline & $\mathrm{CF}_{4}$ & 53.404 & 53.434 & 0.029 & 0.055 \\
\hline & $\mathrm{CO}$ & -13.202 & -13.171 & 0.031 & 0.234 \\
\hline \multirow[t]{5}{*}{${ }^{15} \mathrm{~N}$} & $\mathrm{~N}_{2}$ & -81.592 & -81.541 & 0.052 & 0.063 \\
\hline & $\mathrm{NH}_{3}$ & 275.845 & 275.768 & 0.076 & 0.028 \\
\hline & NNO & -1.517 & -1.503 & 0.014 & 0.930 \\
\hline & $\underline{\mathrm{NNO}}$ & 94.444 & 94.474 & 0.031 & 0.032 \\
\hline & $\overline{\mathrm{PN}}$ & -401.714 & -401.752 & 0.038 & 0.009 \\
\hline \multirow[t]{6}{*}{${ }^{17} \mathrm{O}$} & furan & 37.547 & 37.438 & 0.109 & 0.290 \\
\hline & $\left(\mathrm{CH}_{3}\right)_{2} \mathrm{CO}$ & -316.519 & -316.779 & 0.260 & 0.082 \\
\hline & $\mathrm{H}_{2} \mathrm{O}$ & 349.495 & 349.468 & 0.027 & 0.008 \\
\hline & $\mathrm{CO}$ & -64.958 & -64.977 & 0.019 & 0.029 \\
\hline & $\mathrm{OF}_{2}$ & -538.323 & -538.433 & 0.110 & 0.020 \\
\hline & NNO & 191.339 & 191.329 & 0.010 & 0.005 \\
\hline \multirow[t]{5}{*}{${ }^{19} \mathrm{~F}$} & $\mathrm{HF}$ & 437.037 & 437.044 & 0.007 & 0.002 \\
\hline & $\mathrm{F}_{2}$ & -215.668 & -215.470 & 0.198 & 0.092 \\
\hline & $\mathrm{CF}_{4}$ & 262.882 & 263.046 & 0.165 & 0.063 \\
\hline & $\mathrm{OF}_{2}$ & -34.200 & -34.063 & 0.137 & 0.400 \\
\hline & $\mathrm{PF}_{3}$ & 227.022 & 227.039 & 0.017 & 0.007 \\
\hline \multirow[t]{3}{*}{${ }^{31} \mathrm{P}$} & $\mathrm{PH}_{3}$ & 598.967 & 598.582 & 0.385 & 0.064 \\
\hline & $\mathrm{PF}_{3}$ & 189.238 & 189.124 & 0.114 & 0.060 \\
\hline & $\mathrm{PN}$ & -44.887 & -44.955 & 0.068 & 0.151 \\
\hline \multirow{3}{*}{\multicolumn{3}{|c|}{$\begin{array}{l}\text { MAE/MARE }\left({ }^{1} \mathrm{H}\right) \\
\text { MAE/MARE }\left({ }^{13} \mathrm{C}\right) \\
\text { MAE/MARE (other) }\end{array}$}} & & 0.001 & 0.004 \\
\hline & & & & 0.041 & 0.092 \\
\hline & & & & 0.097 & 0.123 \\
\hline
\end{tabular}


Table S9: Absolute $\Delta \sigma_{a b s}$ (in ppm) and relative deviations $\Delta \sigma_{r e l}$ (in \%) due to P-junction threshold $\left(10^{-j X}\right.$, with $j X=2,3,4,5)$ for the Lh07t-SVWN functional

\begin{tabular}{|c|c|c|c|c|c|c|c|c|c|c|}
\hline \multicolumn{3}{|c|}{ P-junctions } & \multicolumn{4}{|c|}{$\Delta \sigma_{a b s}[\mathrm{ppm}]$} & \multicolumn{4}{|c|}{$\Delta \sigma_{r e l}[\%]$} \\
\hline & molecule & no screening & $\mathrm{j} 2$ & j3 & $\mathrm{j} 4$ & $\mathrm{j} 5$ & $\mathrm{j} 2$ & j3 & $\mathrm{j} 4$ & $\mathrm{j} 5$ \\
\hline \multirow[t]{8}{*}{${ }^{1} \mathrm{H}$} & furan $(\mathrm{C} 2 / 5)$ & 23.681 & 0.029 & 0.001 & 0.000 & 0.000 & 0.121 & 0.005 & 0.000 & 0.000 \\
\hline & furan (C3/4) & 24.626 & 0.125 & 0.001 & 0.000 & 0.000 & 0.508 & 0.006 & 0.000 & 0.000 \\
\hline & $\left(\mathrm{CH}_{3}\right)_{2} \mathrm{CO}$ & 29.283 & 0.071 & 0.000 & 0.000 & 0.000 & 0.243 & 0.001 & 0.000 & 0.000 \\
\hline & $\mathrm{CH}_{4}$ & 31.307 & 0.100 & 0.003 & 0.000 & 0.000 & 0.318 & 0.009 & 0.000 & 0.000 \\
\hline & $\mathrm{NH}_{3}$ & 31.326 & 0.274 & 0.011 & 0.000 & 0.000 & 0.874 & 0.036 & 0.001 & 0.000 \\
\hline & $\mathrm{H}_{2} \mathrm{O}$ & 30.474 & 0.035 & 0.004 & 0.000 & 0.000 & 0.113 & 0.012 & 0.000 & 0.000 \\
\hline & $\mathrm{HF}$ & 28.552 & 0.172 & 0.004 & 0.000 & 0.000 & 0.603 & 0.013 & 0.000 & 0.000 \\
\hline & $\mathrm{PH}_{3}$ & 29.251 & 0.010 & 0.004 & 0.000 & 0.000 & 0.035 & 0.012 & 0.000 & 0.000 \\
\hline \multirow[t]{7}{*}{${ }^{13} \mathrm{C}$} & furan $(\mathrm{C} 2 / 5)$ & 38.015 & 2.193 & 0.048 & 0.001 & 0.000 & 5.770 & 0.127 & 0.002 & 0.000 \\
\hline & furan $(\mathrm{C} 3 / 4)$ & 72.317 & 1.473 & 0.038 & 0.001 & 0.000 & 2.036 & 0.053 & 0.001 & 0.000 \\
\hline & $\left(\mathrm{CH}_{3}\right)_{2} \underline{\mathrm{CO}}$ & -30.312 & 0.201 & 0.028 & 0.001 & 0.000 & 0.663 & 0.093 & 0.002 & 0.000 \\
\hline & $\left(\underline{\mathrm{CH}}_{3}\right)_{2} \overline{\mathrm{CO}}$ & 159.382 & 2.807 & 0.072 & 0.001 & 0.000 & 1.761 & 0.045 & 0.001 & 0.000 \\
\hline & $\overline{\mathrm{CH}}_{4}$ & 199.852 & 11.619 & 0.286 & 0.005 & 0.000 & 5.814 & 0.143 & 0.002 & 0.000 \\
\hline & $\mathrm{CF}_{4}$ & 53.404 & 0.193 & 0.004 & 0.000 & 0.000 & 0.361 & 0.008 & 0.000 & 0.000 \\
\hline & $\mathrm{CO}$ & -13.202 & 1.473 & 0.016 & 0.000 & 0.000 & 11.154 & 0.119 & 0.000 & 0.000 \\
\hline \multirow[t]{5}{*}{${ }^{15} \mathrm{~N}$} & $\mathrm{~N}_{2}$ & -81.593 & 1.324 & 0.027 & 0.000 & 0.000 & 1.622 & 0.034 & 0.000 & 0.000 \\
\hline & $\mathrm{NH}_{3}$ & 275.845 & 15.380 & 0.652 & 0.013 & 0.000 & 5.575 & 0.236 & 0.005 & 0.000 \\
\hline & $\mathrm{NNO}$ & -1.517 & 0.733 & 0.016 & 0.000 & 0.000 & 48.335 & 1.083 & 0.005 & 0.000 \\
\hline & $\underline{N N O}$ & 94.444 & 1.599 & 0.041 & 0.001 & 0.000 & 1.694 & 0.043 & 0.001 & 0.000 \\
\hline & $\overline{\mathrm{PN}}$ & -401.714 & 6.618 & 0.122 & 0.001 & 0.000 & 1.647 & 0.030 & 0.000 & 0.000 \\
\hline \multirow[t]{6}{*}{${ }^{17} \mathrm{O}$} & furan & 37.547 & 2.488 & 0.023 & 0.000 & 0.000 & 6.625 & 0.062 & 0.001 & 0.000 \\
\hline & $\left(\mathrm{CH}_{3}\right)_{2} \mathrm{CO}$ & -316.520 & 8.913 & 0.344 & 0.007 & 0.000 & 2.816 & 0.109 & 0.002 & 0.000 \\
\hline & $\mathrm{H}_{2} \mathrm{O}$ & 349.495 & 17.524 & 0.683 & 0.014 & 0.000 & 5.014 & 0.195 & 0.004 & 0.000 \\
\hline & $\mathrm{CO}$ & -64.958 & 3.973 & 0.075 & 0.001 & 0.000 & 6.117 & 0.115 & 0.001 & 0.000 \\
\hline & $\mathrm{OF}_{2}$ & -538.323 & 1.214 & 0.021 & 0.000 & 0.000 & 0.226 & 0.004 & 0.000 & 0.000 \\
\hline & NNO & 191.339 & 3.931 & 0.129 & 0.003 & 0.000 & 2.055 & 0.067 & 0.002 & 0.000 \\
\hline \multirow[t]{5}{*}{${ }^{19} \mathrm{~F}$} & $\mathrm{HF}$ & 437.037 & 11.054 & 0.450 & 0.010 & 0.000 & 2.529 & 0.103 & 0.002 & 0.000 \\
\hline & $\mathrm{F}_{2}$ & -215.668 & 1.736 & 0.043 & 0.001 & 0.000 & 0.805 & 0.020 & 0.000 & 0.000 \\
\hline & $\mathrm{CF}_{4}$ & 262.882 & 1.344 & 0.055 & 0.001 & 0.000 & 0.511 & 0.021 & 0.000 & 0.000 \\
\hline & $\mathrm{OF}_{2}$ & -34.200 & 0.021 & 0.005 & 0.000 & 0.000 & 0.061 & 0.014 & 0.000 & 0.000 \\
\hline & $\mathrm{PF}_{3}$ & 227.022 & 1.203 & 0.022 & 0.000 & 0.000 & 0.530 & 0.009 & 0.000 & 0.000 \\
\hline \multirow[t]{3}{*}{${ }^{31} \mathrm{P}$} & $\mathrm{PH}_{3}$ & 598.967 & 34.570 & 0.930 & 0.016 & 0.000 & 5.772 & 0.155 & 0.003 & 0.000 \\
\hline & $\mathrm{PF}_{3}$ & 189.238 & 1.002 & 0.051 & 0.001 & 0.000 & 0.529 & 0.027 & 0.000 & 0.000 \\
\hline & $\mathrm{PN}$ & -44.886 & 9.981 & 0.174 & 0.003 & 0.000 & 22.238 & 0.387 & 0.006 & 0.000 \\
\hline \multirow{3}{*}{\multicolumn{2}{|c|}{$\begin{array}{l}\text { MAE/MARE }\left({ }^{1} \mathrm{H}\right) \\
\text { MAE/MARE }\left({ }^{13} \mathrm{C}\right) \\
\text { MAE/MARE (other) }\end{array}$}} & & 0.102 & 0.003 & 0.000 & 0.000 & 0.352 & 0.012 & 0.000 & 0.000 \\
\hline & & & 2.851 & 0.070 & 0.001 & 0.000 & 3.937 & 0.084 & 0.001 & 0.000 \\
\hline & & & 6.558 & 0.203 & 0.004 & 0.000 & 6.037 & 0.143 & 0.002 & 0.000 \\
\hline
\end{tabular}


Table S10: Absolute $\Delta \sigma_{a b s}$ (in ppm) and relative deviations $\Delta \sigma_{\text {rel }}$ (in \%) due to S-junction threshold (10 ${ }^{-j X}$, with $j X=3,4,5)$ for the Lh07t-SVWN functional

\begin{tabular}{|c|c|c|c|c|c|c|c|c|}
\hline \multicolumn{3}{|c|}{ S-junctions } & \multicolumn{3}{|c|}{$\Delta \sigma_{a b s}[\mathrm{ppm}]$} & \multicolumn{3}{|c|}{$\Delta \sigma_{\text {rel }}[\%]$} \\
\hline & molecule & no screening & j3 & $\mathrm{j} 4$ & $\mathrm{j} 5$ & j3 & $\mathrm{j} 4$ & $\mathrm{j} 5$ \\
\hline \multirow[t]{8}{*}{${ }^{1} \mathrm{H}$} & furan $(\mathrm{C} 2 / 5)$ & 23.681 & 0.000 & 0.000 & 0.000 & 0.000 & 0.000 & 0.000 \\
\hline & furan (C3/4) & 24.626 & 0.001 & 0.000 & 0.000 & 0.003 & 0.000 & 0.000 \\
\hline & $\left(\mathrm{CH}_{3}\right)_{2} \mathrm{CO}$ & 29.283 & 0.000 & 0.000 & 0.000 & 0.001 & 0.000 & 0.000 \\
\hline & $\mathrm{CH}_{4}$ & 31.307 & 0.000 & 0.000 & 0.000 & 0.000 & 0.000 & 0.000 \\
\hline & $\mathrm{NH}_{3}$ & 31.326 & 0.000 & 0.000 & 0.000 & 0.000 & 0.000 & 0.000 \\
\hline & $\mathrm{H}_{2} \mathrm{O}$ & 30.474 & 0.000 & 0.000 & 0.000 & 0.000 & 0.000 & 0.000 \\
\hline & $\mathrm{HF}$ & 28.552 & 0.000 & 0.000 & 0.000 & 0.000 & 0.000 & 0.000 \\
\hline & $\mathrm{PH}_{3}$ & 29.251 & 0.000 & 0.000 & 0.000 & 0.000 & 0.000 & 0.000 \\
\hline \multirow[t]{7}{*}{${ }^{13} \mathrm{C}$} & furan $(\mathrm{C} 2 / 5)$ & 38.015 & 0.006 & 0.000 & 0.000 & 0.017 & 0.000 & 0.000 \\
\hline & furan $(\mathrm{C} 3 / 4)$ & 72.317 & 0.004 & 0.001 & 0.000 & 0.005 & 0.001 & 0.000 \\
\hline & $\left(\mathrm{CH}_{3}\right)_{2} \underline{\mathrm{CO}}$ & -30.312 & 0.001 & 0.000 & 0.000 & 0.005 & 0.001 & 0.000 \\
\hline & $\left(\mathrm{CH}_{3}\right)_{2} \mathrm{CO}$ & 159.382 & 0.001 & 0.000 & 0.000 & 0.001 & 0.000 & 0.000 \\
\hline & $\mathrm{CH}_{4}$ & 199.852 & 0.001 & 0.000 & 0.000 & 0.001 & 0.000 & 0.000 \\
\hline & $\mathrm{CF}_{4}$ & 53.404 & 0.114 & 0.006 & 0.000 & 0.213 & 0.010 & 0.000 \\
\hline & $\mathrm{CO}$ & -13.202 & 0.039 & 0.000 & 0.000 & 0.297 & 0.003 & 0.001 \\
\hline \multirow[t]{5}{*}{${ }^{15} \mathrm{~N}$} & $\mathrm{~N}_{2}$ & -81.593 & 0.038 & 0.038 & 0.000 & 0.047 & 0.047 & 0.000 \\
\hline & $\mathrm{NH}_{3}$ & 275.845 & 0.000 & 0.000 & 0.000 & 0.000 & 0.000 & 0.000 \\
\hline & NNO & -1.517 & 0.019 & 0.010 & 0.000 & 1.248 & 0.676 & 0.006 \\
\hline & $\underline{\mathrm{NNO}}$ & 94.444 & 0.020 & 0.010 & 0.000 & 0.021 & 0.011 & 0.000 \\
\hline & $\mathrm{PN}$ & -401.714 & 0.002 & 0.001 & 0.000 & 0.000 & 0.000 & 0.000 \\
\hline \multirow[t]{6}{*}{${ }^{17} \mathrm{O}$} & furan & 37.547 & 0.085 & 0.001 & 0.000 & 0.226 & 0.002 & 0.000 \\
\hline & $\left(\mathrm{CH}_{3}\right)_{2} \mathrm{CO}$ & -316.520 & 0.000 & 0.000 & 0.000 & 0.000 & 0.000 & 0.000 \\
\hline & $\mathrm{H}_{2} \mathrm{O}$ & 349.495 & 0.000 & 0.000 & 0.000 & 0.000 & 0.000 & 0.000 \\
\hline & $\mathrm{CO}$ & -64.958 & 0.094 & 0.001 & 0.000 & 0.145 & 0.001 & 0.001 \\
\hline & $\mathrm{OF}_{2}$ & -538.323 & 0.043 & 0.000 & 0.000 & 0.008 & 0.000 & 0.000 \\
\hline & NNO & 191.339 & 0.017 & 0.013 & 0.000 & 0.009 & 0.007 & 0.000 \\
\hline \multirow[t]{5}{*}{${ }^{19} \mathrm{~F}$} & $\mathrm{HF}$ & 437.037 & 0.000 & 0.000 & 0.000 & 0.000 & 0.000 & 0.000 \\
\hline & $\mathrm{F}_{2}$ & -215.668 & 0.042 & 0.017 & 0.000 & 0.020 & 0.008 & 0.000 \\
\hline & $\mathrm{CF}_{4}$ & 262.882 & 0.031 & 0.005 & 0.000 & 0.012 & 0.002 & 0.000 \\
\hline & $\mathrm{OF}_{2}$ & -34.200 & 0.024 & 0.003 & 0.000 & 0.069 & 0.009 & 0.000 \\
\hline & $\mathrm{PF}_{3}$ & 227.022 & 0.010 & 0.000 & 0.000 & 0.004 & 0.000 & 0.000 \\
\hline \multirow[t]{3}{*}{${ }^{31} \mathrm{P}$} & $\mathrm{PH}_{3}$ & 598.967 & 0.012 & 0.000 & 0.000 & 0.002 & 0.000 & 0.000 \\
\hline & $\mathrm{PF}_{3}$ & 189.238 & 0.010 & 0.001 & 0.000 & 0.005 & 0.000 & 0.000 \\
\hline & $\mathrm{PN}$ & -44.886 & 0.005 & 0.001 & 0.000 & 0.011 & 0.003 & 0.000 \\
\hline \multirow{3}{*}{\multicolumn{2}{|c|}{$\begin{array}{l}\text { MAE/MARE }\left({ }^{1} \mathrm{H}\right) \\
\text { MAE/MARE }\left({ }^{13} \mathrm{C}\right) \\
\text { MAE/MARE (other) }\end{array}$}} & & 0.000 & 0.000 & 0.000 & 0.001 & 0.000 & 0.000 \\
\hline & & & 0.024 & 0.001 & 0.000 & 0.077 & 0.002 & 0.000 \\
\hline & & & 0.024 & 0.005 & 0.000 & 0.096 & 0.040 & 0.000 \\
\hline
\end{tabular}


Table S11: Absolute $\Delta \sigma_{a b s}$ (in ppm) and relative deviations $\Delta \sigma_{r e l}$ (in \%) due to PL-junction threshold $\left(10^{-j X}\right.$, with $j X=4,5,6,7)$ for the Lh07t-SVWN functional

\begin{tabular}{|c|c|c|c|c|c|c|c|c|c|c|}
\hline \multicolumn{3}{|c|}{ PL-Junctions } & \multicolumn{4}{|c|}{$\Delta \sigma_{a b s}[\mathrm{ppm}]$} & \multicolumn{4}{|c|}{$\Delta \sigma_{r e l}[\%]$} \\
\hline & molecule & no screening & $\mathrm{j} 4$ & j5 & $\mathrm{j} 6$ & j7 & $\mathrm{j} 4$ & j5 & j6 & j7 \\
\hline \multirow[t]{8}{*}{${ }^{1} \mathrm{H}$} & furan $(\mathrm{C} 2 / 5)$ & 23.681 & 0.013 & 0.006 & 0.000 & 0.000 & 0.054 & 0.027 & 0.001 & 0.000 \\
\hline & furan $(\mathrm{C} 3 / 4)$ & 24.626 & 0.036 & 0.005 & 0.000 & 0.000 & 0.146 & 0.019 & 0.001 & 0.000 \\
\hline & $\left(\mathrm{CH}_{3}\right)_{2} \mathrm{CO}$ & 29.283 & 0.003 & 0.004 & 0.000 & 0.000 & 0.010 & 0.012 & 0.001 & 0.000 \\
\hline & $\mathrm{CH}_{4}$ & 31.307 & 0.022 & 0.006 & 0.000 & 0.000 & 0.070 & 0.018 & 0.001 & 0.000 \\
\hline & $\mathrm{NH}_{3}$ & 31.326 & 0.010 & 0.010 & 0.001 & 0.000 & 0.033 & 0.033 & 0.002 & 0.000 \\
\hline & $\mathrm{H}_{2} \mathrm{O}$ & 30.474 & 0.031 & 0.009 & 0.001 & 0.000 & 0.101 & 0.031 & 0.003 & 0.000 \\
\hline & $\mathrm{HF}$ & 28.552 & 0.001 & 0.003 & 0.002 & 0.000 & 0.003 & 0.011 & 0.008 & 0.000 \\
\hline & $\mathrm{PH}_{3}$ & 29.251 & 0.013 & 0.009 & 0.001 & 0.000 & 0.046 & 0.032 & 0.002 & 0.000 \\
\hline \multirow[t]{7}{*}{${ }^{13} \mathrm{C}$} & furan $(\mathrm{C} 2 / 5)$ & 38.015 & 0.131 & 0.007 & 0.000 & 0.000 & 0.345 & 0.018 & 0.001 & 0.000 \\
\hline & furan $(\mathrm{C} 3 / 4)$ & 72.317 & 0.068 & 0.018 & 0.001 & 0.000 & 0.094 & 0.025 & 0.001 & 0.000 \\
\hline & $\left(\mathrm{CH}_{3}\right)_{2} \underline{\mathrm{CO}}$ & -30.312 & 1.058 & 0.022 & 0.000 & 0.000 & 3.492 & 0.071 & 0.001 & 0.000 \\
\hline & $\left(\mathrm{CH}_{3}\right)_{2} \overline{\mathrm{CO}}$ & 159.382 & 1.377 & 0.080 & 0.002 & 0.000 & 0.864 & 0.050 & 0.001 & 0.000 \\
\hline & $\mathrm{CH}_{4}$ & 199.852 & 4.505 & 0.295 & 0.009 & 0.000 & 2.254 & 0.148 & 0.005 & 0.000 \\
\hline & $\mathrm{CF}_{4}$ & 53.404 & 2.330 & 0.057 & 0.002 & 0.000 & 4.364 & 0.107 & 0.004 & 0.000 \\
\hline & $\mathrm{CO}$ & -13.202 & 1.287 & 0.001 & 0.002 & 0.000 & 9.751 & 0.005 & 0.017 & 0.000 \\
\hline \multirow[t]{5}{*}{${ }^{15} \mathrm{~N}$} & $\mathrm{~N}_{2}$ & -81.593 & 2.025 & 0.005 & 0.002 & 0.000 & 2.482 & 0.006 & 0.002 & 0.000 \\
\hline & $\mathrm{NH}_{3}$ & 275.845 & 5.536 & 0.613 & 0.023 & 0.000 & 2.007 & 0.222 & 0.008 & 0.000 \\
\hline & NNO & -1.517 & 1.717 & 0.037 & 0.000 & 0.000 & 113.170 & 2.433 & 0.002 & 0.002 \\
\hline & $\underline{\mathrm{NNO}}$ & 94.444 & 1.231 & 0.094 & 0.006 & 0.000 & 1.303 & 0.100 & 0.007 & 0.000 \\
\hline & $\mathrm{PN}$ & -401.714 & 0.052 & 0.271 & 0.011 & 0.000 & 0.013 & 0.067 & 0.003 & 0.000 \\
\hline \multirow[t]{6}{*}{${ }^{17} \mathrm{O}$} & furan & 37.547 & 0.093 & 0.009 & 0.000 & 0.000 & 0.248 & 0.023 & 0.000 & 0.000 \\
\hline & $\left(\mathrm{CH}_{3}\right)_{2} \mathrm{CO}$ & -316.520 & 3.894 & 0.335 & 0.011 & 0.000 & 1.230 & 0.106 & 0.004 & 0.000 \\
\hline & $\mathrm{H}_{2} \mathrm{O}$ & 349.495 & 6.173 & 0.926 & 0.035 & 0.001 & 1.766 & 0.265 & 0.010 & 0.000 \\
\hline & $\mathrm{CO}$ & -64.958 & 3.873 & 0.464 & 0.017 & 0.000 & 5.963 & 0.714 & 0.026 & 0.000 \\
\hline & $\mathrm{OF}_{2}$ & -538.323 & 3.679 & 0.114 & 0.006 & 0.000 & 0.683 & 0.021 & 0.001 & 0.000 \\
\hline & NNO & 191.339 & 2.792 & 0.270 & 0.012 & 0.000 & 1.459 & 0.141 & 0.006 & 0.000 \\
\hline \multirow[t]{5}{*}{${ }^{19} \mathrm{~F}$} & $\mathrm{HF}$ & 437.037 & 1.293 & 1.103 & 0.056 & 0.002 & 0.296 & 0.252 & 0.013 & 0.000 \\
\hline & $\mathrm{F}_{2}$ & -215.668 & 12.538 & 0.024 & 0.008 & 0.000 & 5.814 & 0.011 & 0.004 & 0.000 \\
\hline & $\mathrm{CF}_{4}$ & 262.882 & 0.737 & 0.059 & 0.003 & 0.000 & 0.280 & 0.023 & 0.001 & 0.000 \\
\hline & $\mathrm{OF}_{2}$ & -34.200 & 8.912 & 0.108 & 0.002 & 0.000 & 26.059 & 0.315 & 0.007 & 0.000 \\
\hline & $\mathrm{PF}_{3}$ & 227.022 & 2.556 & 0.121 & 0.003 & 0.000 & 1.126 & 0.053 & 0.001 & 0.000 \\
\hline \multirow[t]{3}{*}{${ }^{31} \mathrm{P}$} & $\mathrm{PH}_{3}$ & 598.967 & 8.022 & 0.783 & 0.021 & 0.000 & 1.339 & 0.131 & 0.003 & 0.000 \\
\hline & $\mathrm{PF}_{3}$ & 189.238 & 2.552 & 0.111 & 0.000 & 0.000 & 1.348 & 0.059 & 0.000 & 0.000 \\
\hline & $\mathrm{PN}$ & -44.886 & 7.083 & 0.754 & 0.027 & 0.001 & 15.781 & 1.681 & 0.061 & 0.002 \\
\hline \multicolumn{3}{|c|}{ MAE/MARE $\left({ }^{1} \mathrm{H}\right)$} & 0.016 & 0.007 & 0.001 & 0.000 & 0.058 & 0.023 & 0.003 & 0.000 \\
\hline \multicolumn{3}{|c|}{ MAE/MARE $\left({ }^{13} \mathrm{C}\right)$} & 1.537 & 0.069 & 0.002 & 0.000 & 3.023 & 0.061 & 0.004 & 0.000 \\
\hline \multicolumn{3}{|c|}{ MAE/MARE (other) } & 3.935 & 0.326 & 0.013 & 0.000 & 9.598 & 0.349 & 0.008 & 0.000 \\
\hline
\end{tabular}


Table S12: Absolute $\Delta \sigma_{a b s}$ (in ppm) due to F-junction threshold $\left(10^{-j X}\right.$, with $\left.j X=4,5,6,7,8,9\right)$ for the Lh07tSVWN functional

\begin{tabular}{|c|c|c|c|c|c|c|c|c|}
\hline \multicolumn{3}{|c|}{ F-Junctions } & \multicolumn{6}{|c|}{$\Delta \sigma_{a b s}[\mathrm{ppm}]$} \\
\hline & molecule & no screening & $\mathrm{j} 4$ & j5 & $\mathrm{j} 6$ & j7 & j8 & j9 \\
\hline \multirow[t]{8}{*}{$\overline{{ }^{1} \mathrm{H}}$} & furan $(\mathrm{C} 2 / 5)$ & 23.681 & 0.059 & 0.080 & 0.028 & 0.006 & 0.001 & 0.000 \\
\hline & furan $(\mathrm{C} 3 / 4)$ & 24.626 & 0.092 & 0.029 & 0.010 & 0.002 & 0.000 & 0.000 \\
\hline & $\left(\mathrm{CH}_{3}\right)_{2} \mathrm{CO}$ & 29.283 & 0.007 & 0.022 & 0.012 & 0.003 & 0.001 & 0.000 \\
\hline & $\mathrm{CH}_{4}$ & 31.307 & 0.106 & 0.020 & 0.005 & 0.001 & 0.000 & 0.000 \\
\hline & $\mathrm{NH}_{3}$ & 31.326 & 0.073 & 0.011 & 0.002 & 0.001 & 0.000 & 0.000 \\
\hline & $\mathrm{H}_{2} \mathrm{O}$ & 30.474 & 0.030 & 0.006 & 0.001 & 0.000 & 0.000 & 0.000 \\
\hline & $\mathrm{HF}$ & 28.552 & 0.006 & 0.000 & 0.000 & 0.000 & 0.000 & 0.000 \\
\hline & $\mathrm{PH}_{3}$ & 29.251 & 0.009 & 0.001 & 0.000 & 0.000 & 0.000 & 0.000 \\
\hline \multirow[t]{7}{*}{${ }^{13} \mathrm{C}$} & furan $(\mathrm{C} 2 / 5)$ & 38.015 & 0.955 & 0.269 & 0.080 & 0.018 & 0.005 & 0.001 \\
\hline & furan (C3/4) & 72.317 & 0.124 & 0.077 & 0.018 & 0.005 & 0.002 & 0.000 \\
\hline & $\left(\mathrm{CH}_{3}\right)_{2} \underline{\mathrm{CO}}$ & -30.312 & 1.053 & 0.293 & 0.066 & 0.018 & 0.005 & 0.001 \\
\hline & $\left(\underline{\mathrm{CH}}_{3}\right)_{2} \overline{\mathrm{CO}}$ & 159.382 & 0.502 & 0.144 & 0.064 & 0.016 & 0.003 & 0.001 \\
\hline & $\overline{\mathrm{CH}}_{4}$ & 199.852 & 3.865 & 0.812 & 0.185 & 0.036 & 0.007 & 0.002 \\
\hline & $\mathrm{CF}_{4}$ & 53.404 & 0.365 & 0.044 & 0.010 & 0.008 & 0.001 & 0.000 \\
\hline & $\mathrm{CO}$ & -13.202 & 0.384 & 0.110 & 0.023 & 0.005 & 0.001 & 0.000 \\
\hline \multirow[t]{5}{*}{${ }^{15} \mathrm{~N}$} & $\mathrm{~N}_{2}$ & -81.593 & 0.413 & 0.101 & 0.020 & 0.004 & 0.001 & 0.000 \\
\hline & $\mathrm{NH}_{3}$ & 275.845 & 1.407 & 0.232 & 0.055 & 0.012 & 0.002 & 0.001 \\
\hline & NNO & -1.517 & 0.251 & 0.078 & 0.018 & 0.005 & 0.001 & 0.000 \\
\hline & $\underline{\mathrm{NNO}}$ & 94.444 & 0.048 & 0.063 & 0.032 & 0.007 & 0.001 & 0.000 \\
\hline & $\overline{\mathrm{PN}}$ & -401.714 & 0.402 & 0.084 & 0.059 & 0.014 & 0.003 & 0.001 \\
\hline \multirow[t]{6}{*}{${ }^{17} \mathrm{O}$} & furan & 37.547 & 4.086 & 1.672 & 0.421 & 0.090 & 0.018 & 0.003 \\
\hline & $\left(\mathrm{CH}_{3}\right)_{2} \mathrm{CO}$ & -316.520 & 2.040 & 0.993 & 0.360 & 0.084 & 0.020 & 0.004 \\
\hline & $\mathrm{H}_{2} \mathrm{O}$ & 349.495 & 1.120 & 0.180 & 0.040 & 0.009 & 0.002 & 0.000 \\
\hline & $\mathrm{CO}$ & -64.958 & 0.338 & 0.021 & 0.004 & 0.001 & 0.000 & 0.000 \\
\hline & $\mathrm{OF}_{2}$ & -538.323 & 2.409 & 0.554 & 0.097 & 0.021 & 0.004 & 0.001 \\
\hline & NNO & 191.339 & 1.318 & 0.374 & 0.048 & 0.012 & 0.002 & 0.000 \\
\hline \multirow[t]{5}{*}{${ }^{19} \mathrm{~F}$} & $\mathrm{HF}$ & 437.037 & 0.168 & 0.044 & 0.011 & 0.003 & 0.001 & 0.000 \\
\hline & $\mathrm{F}_{2}$ & -215.668 & 0.175 & 0.055 & 0.027 & 0.007 & 0.001 & 0.000 \\
\hline & $\mathrm{CF}_{4}$ & 262.882 & 0.798 & 0.110 & 0.009 & 0.001 & 0.001 & 0.000 \\
\hline & $\mathrm{OF}_{2}$ & -34.200 & 0.117 & 0.276 & 0.082 & 0.018 & 0.004 & 0.001 \\
\hline & $\mathrm{PF}_{3}$ & 227.022 & 0.306 & 0.135 & 0.078 & 0.015 & 0.003 & 0.001 \\
\hline \multirow[t]{3}{*}{${ }^{31} \mathrm{P}$} & $\mathrm{PH}_{3}$ & 598.967 & 7.334 & 2.027 & 0.394 & 0.064 & 0.014 & 0.003 \\
\hline & $\mathrm{PF}_{3}$ & 189.238 & 0.688 & 0.906 & 0.386 & 0.080 & 0.016 & 0.004 \\
\hline & $\mathrm{PN}$ & -44.886 & 0.548 & 0.050 & 0.076 & 0.019 & 0.003 & 0.001 \\
\hline \multicolumn{2}{|c|}{ MAE $\left({ }^{1} \mathrm{H}\right)$} & & 0.048 & 0.021 & 0.007 & 0.002 & 0.000 & 0.000 \\
\hline \multicolumn{2}{|c|}{ MAE $\left({ }^{13} \mathrm{C}\right)$} & & 1.035 & 0.250 & 0.064 & 0.015 & 0.003 & 0.001 \\
\hline \multicolumn{2}{|c|}{ MAE (other) } & & 1.261 & 0.419 & 0.117 & 0.025 & 0.005 & 0.001 \\
\hline
\end{tabular}


Table S13: Relative deviations $\Delta \sigma_{r e l}$ (in \%) due to F-junction threshold $\left(10^{-j X}\right.$, with $\left.j X=4,5,6,7,8,9\right)$ for the Lh07t-SVWN functional

\begin{tabular}{|c|c|c|c|c|c|c|c|c|}
\hline \multicolumn{3}{|c|}{ F-Junctions } & \multicolumn{6}{|c|}{$\Delta \sigma_{\text {rel }}[\%]$} \\
\hline & molecule & no screening & j4 & $\mathrm{j} 5$ & j6 & j7 & j8 & j9 \\
\hline \multirow[t]{8}{*}{${ }^{1} \mathrm{H}$} & furan $(\mathrm{C} 2 / 5)$ & 23.681 & 0.249 & 0.336 & 0.117 & 0.024 & 0.006 & 0.001 \\
\hline & furan $(\mathrm{C} 3 / 4)$ & 24.626 & 0.374 & 0.119 & 0.043 & 0.009 & 0.002 & 0.000 \\
\hline & $\left(\mathrm{CH}_{3}\right)_{2} \mathrm{CO}$ & 29.283 & 0.022 & 0.076 & 0.040 & 0.010 & 0.002 & 0.001 \\
\hline & $\mathrm{CH}_{4}$ & 31.307 & 0.337 & 0.064 & 0.017 & 0.003 & 0.001 & 0.000 \\
\hline & $\mathrm{NH}_{3}$ & 31.326 & 0.234 & 0.034 & 0.008 & 0.002 & 0.000 & 0.000 \\
\hline & $\mathrm{H}_{2} \mathrm{O}$ & 30.474 & 0.098 & 0.019 & 0.004 & 0.001 & 0.000 & 0.000 \\
\hline & $\mathrm{HF}$ & 28.552 & 0.021 & 0.001 & 0.001 & 0.001 & 0.000 & 0.000 \\
\hline & $\mathrm{PH}_{3}$ & 29.251 & 0.030 & 0.002 & 0.001 & 0.001 & 0.000 & 0.000 \\
\hline \multirow[t]{7}{*}{${ }^{13} \mathrm{C}$} & furan $(\mathrm{C} 2 / 5)$ & 38.015 & 2.512 & 0.708 & 0.210 & 0.046 & 0.013 & 0.003 \\
\hline & furan (C3/4) & 72.317 & 0.172 & 0.107 & 0.025 & 0.007 & 0.003 & 0.000 \\
\hline & $\left(\mathrm{CH}_{3}\right)_{2} \underline{\mathrm{CO}}$ & -30.312 & 3.474 & 0.965 & 0.218 & 0.058 & 0.016 & 0.003 \\
\hline & $\left(\underline{\mathrm{CH}}_{3}\right)_{2} \overline{\mathrm{CO}}$ & 159.382 & 0.315 & 0.090 & 0.040 & 0.010 & 0.002 & 0.001 \\
\hline & $\overline{\mathrm{CH}}_{4}$ & 199.852 & 1.934 & 0.406 & 0.093 & 0.018 & 0.004 & 0.001 \\
\hline & $\mathrm{CF}_{4}$ & 53.404 & 0.683 & 0.083 & 0.019 & 0.015 & 0.002 & 0.000 \\
\hline & $\mathrm{CO}$ & -13.202 & 2.912 & 0.834 & 0.175 & 0.035 & 0.008 & 0.002 \\
\hline \multirow[t]{5}{*}{${ }^{15} \mathrm{~N}$} & $\mathrm{~N}_{2}$ & -81.593 & 0.507 & 0.124 & 0.024 & 0.005 & 0.001 & 0.000 \\
\hline & $\mathrm{NH}_{3}$ & 275.845 & 0.510 & 0.084 & 0.020 & 0.004 & 0.001 & 0.000 \\
\hline & NNO & -1.517 & 16.538 & 5.147 & 1.165 & 0.347 & 0.059 & 0.014 \\
\hline & $\underline{\mathrm{NNO}}$ & 94.444 & 0.051 & 0.067 & 0.033 & 0.007 & 0.001 & 0.000 \\
\hline & $\overline{\mathrm{PN}}$ & -401.714 & 0.100 & 0.021 & 0.015 & 0.003 & 0.001 & 0.000 \\
\hline \multirow[t]{6}{*}{${ }^{17} \mathrm{O}$} & furan & 37.547 & 10.882 & 4.452 & 1.120 & 0.239 & 0.047 & 0.009 \\
\hline & $\left(\mathrm{CH}_{3}\right)_{2} \mathrm{CO}$ & -316.520 & 0.645 & 0.314 & 0.114 & 0.027 & 0.006 & 0.001 \\
\hline & $\mathrm{H}_{2} \mathrm{O}$ & 349.495 & 0.320 & 0.052 & 0.011 & 0.003 & 0.000 & 0.000 \\
\hline & $\mathrm{CO}$ & -64.958 & 0.520 & 0.032 & 0.007 & 0.001 & 0.000 & 0.000 \\
\hline & $\mathrm{OF}_{2}$ & -538.323 & 0.448 & 0.103 & 0.018 & 0.004 & 0.001 & 0.000 \\
\hline & NNO & 191.339 & 0.689 & 0.195 & 0.025 & 0.006 & 0.001 & 0.000 \\
\hline \multirow[t]{5}{*}{${ }^{19} \mathrm{~F}$} & $\mathrm{HF}$ & 437.037 & 0.038 & 0.010 & 0.002 & 0.001 & 0.000 & 0.000 \\
\hline & $\mathrm{F}_{2}$ & -215.668 & 0.081 & 0.026 & 0.013 & 0.003 & 0.001 & 0.000 \\
\hline & $\mathrm{CF}_{4}$ & 262.882 & 0.303 & 0.042 & 0.003 & 0.001 & 0.000 & 0.000 \\
\hline & $\mathrm{OF}_{2}$ & -34.200 & 0.342 & 0.806 & 0.241 & 0.053 & 0.011 & 0.002 \\
\hline & $\mathrm{PF}_{3}$ & 227.022 & 0.135 & 0.059 & 0.034 & 0.006 & 0.001 & 0.000 \\
\hline \multirow[t]{3}{*}{${ }^{31} \mathrm{P}$} & $\mathrm{PH}_{3}$ & 598.967 & 1.224 & 0.338 & 0.066 & 0.011 & 0.002 & 0.001 \\
\hline & $\mathrm{PF}_{3}$ & 189.238 & 0.364 & 0.479 & 0.204 & 0.042 & 0.008 & 0.002 \\
\hline & $\mathrm{PN}$ & -44.886 & 1.220 & 0.110 & 0.169 & 0.043 & 0.008 & 0.002 \\
\hline \multicolumn{3}{|c|}{ MARE $\left({ }^{1} \mathrm{H}\right)$} & 0.171 & 0.081 & 0.029 & 0.006 & 0.001 & 0.000 \\
\hline \multicolumn{3}{|c|}{ MARE $\left({ }^{13} \mathrm{C}\right)$} & 1.714 & 0.456 & 0.112 & 0.027 & 0.007 & 0.001 \\
\hline \multicolumn{3}{|c|}{ MARE (other) } & 1.838 & 0.656 & 0.173 & 0.042 & 0.008 & 0.002 \\
\hline
\end{tabular}


Table S14: Absolute $\Delta \sigma_{a b s}$ (in ppm) and relative deviations $\Delta \sigma_{r e l}$ (in \%) due to FL-junction threshold $\left(10^{-j X}\right.$, with $j X=9,10,11)$ for the Lh07t-SVWN functional

\begin{tabular}{|c|c|c|c|c|c|c|c|c|}
\hline \multicolumn{3}{|c|}{ FL-Junctions } & \multicolumn{3}{|c|}{$\Delta \sigma_{a b s}[\mathrm{ppm}]$} & \multicolumn{3}{|c|}{$\Delta \sigma_{\text {rel }}[\%]$} \\
\hline & molecule & no screening & j9 & j10 & j11 & j9 & j10 & j11 \\
\hline \multirow[t]{8}{*}{${ }^{1} \mathrm{H}$} & furan $(\mathrm{C} 2 / 5)$ & 23.681 & 0.001 & 0.000 & 0.000 & 0.003 & 0.001 & 0.000 \\
\hline & furan $(\mathrm{C} 3 / 4)$ & 24.626 & 0.002 & 0.001 & 0.000 & 0.010 & 0.002 & 0.000 \\
\hline & $\left(\mathrm{CH}_{3}\right)_{2} \mathrm{CO}$ & 29.283 & 0.000 & 0.000 & 0.000 & 0.000 & 0.000 & 0.000 \\
\hline & $\mathrm{CH}_{4}$ & 31.307 & 0.000 & 0.000 & 0.000 & 0.000 & 0.000 & 0.000 \\
\hline & $\mathrm{NH}_{3}$ & 31.326 & 0.000 & 0.000 & 0.000 & 0.000 & 0.000 & 0.000 \\
\hline & $\mathrm{H}_{2} \mathrm{O}$ & 30.474 & 0.000 & 0.000 & 0.000 & 0.001 & 0.000 & 0.000 \\
\hline & $\mathrm{HF}$ & 28.552 & 0.001 & 0.000 & 0.000 & 0.003 & 0.000 & 0.000 \\
\hline & $\mathrm{PH}_{3}$ & 29.251 & 0.000 & 0.000 & 0.000 & 0.001 & 0.001 & 0.000 \\
\hline \multirow[t]{7}{*}{${ }^{13} \mathrm{C}$} & furan $(\mathrm{C} 2 / 5)$ & 38.015 & 0.011 & 0.003 & 0.001 & 0.030 & 0.007 & 0.001 \\
\hline & furan $(\mathrm{C} 3 / 4)$ & 72.317 & 0.008 & 0.002 & 0.000 & 0.011 & 0.003 & 0.001 \\
\hline & $\left(\mathrm{CH}_{3}\right)_{2} \underline{\mathrm{CO}}$ & -30.312 & 0.006 & 0.002 & 0.000 & 0.018 & 0.005 & 0.001 \\
\hline & $\left(\underline{\mathrm{CH}}_{3}\right)_{2} \mathrm{CO}$ & 159.382 & 0.015 & 0.004 & 0.001 & 0.009 & 0.003 & 0.001 \\
\hline & $\overline{\mathrm{CH}}_{4}$ & 199.852 & 0.019 & 0.005 & 0.001 & 0.009 & 0.002 & 0.001 \\
\hline & $\mathrm{CF}_{4}$ & 53.404 & 0.016 & 0.004 & 0.001 & 0.030 & 0.008 & 0.002 \\
\hline & $\mathrm{CO}$ & -13.202 & 0.009 & 0.002 & 0.000 & 0.066 & 0.014 & 0.003 \\
\hline \multirow[t]{5}{*}{${ }^{15} \mathrm{~N}$} & $\mathrm{~N}_{2}$ & -81.593 & 0.000 & 0.000 & 0.000 & 0.000 & 0.000 & 0.000 \\
\hline & $\mathrm{NH}_{3}$ & 275.845 & 0.012 & 0.003 & 0.001 & 0.004 & 0.001 & 0.000 \\
\hline & NNO & -1.517 & 0.003 & 0.001 & 0.000 & 0.207 & 0.075 & 0.013 \\
\hline & $\underline{\mathrm{NNO}}$ & 94.444 & 0.014 & 0.003 & 0.001 & 0.015 & 0.003 & 0.001 \\
\hline & $\mathrm{PN}$ & -401.714 & 0.034 & 0.007 & 0.001 & 0.008 & 0.002 & 0.000 \\
\hline \multirow[t]{6}{*}{${ }^{17} \mathrm{O}$} & furan & 37.547 & 0.020 & 0.003 & 0.001 & 0.052 & 0.009 & 0.002 \\
\hline & $\left(\mathrm{CH}_{3}\right)_{2} \mathrm{CO}$ & -316.520 & 0.052 & 0.012 & 0.003 & 0.017 & 0.004 & 0.001 \\
\hline & $\mathrm{H}_{2} \mathrm{O}$ & 349.495 & 0.006 & 0.001 & 0.000 & 0.002 & 0.000 & 0.000 \\
\hline & $\mathrm{CO}$ & -64.958 & 0.017 & 0.003 & 0.001 & 0.026 & 0.004 & 0.001 \\
\hline & $\mathrm{OF}_{2}$ & -538.323 & 0.068 & 0.012 & 0.003 & 0.013 & 0.002 & 0.001 \\
\hline & NNO & 191.339 & 0.039 & 0.007 & 0.001 & 0.020 & 0.004 & 0.001 \\
\hline \multirow[t]{5}{*}{${ }^{19} \mathrm{~F}$} & $\mathrm{HF}$ & 437.037 & 0.003 & 0.001 & 0.000 & 0.001 & 0.000 & 0.000 \\
\hline & $\mathrm{F}_{2}$ & -215.668 & 0.079 & 0.018 & 0.004 & 0.037 & 0.009 & 0.002 \\
\hline & $\mathrm{CF}_{4}$ & 262.882 & 0.001 & 0.001 & 0.000 & 0.000 & 0.001 & 0.000 \\
\hline & $\mathrm{OF}_{2}$ & -34.200 & 0.046 & 0.010 & 0.002 & 0.135 & 0.030 & 0.006 \\
\hline & $\mathrm{PF}_{3}$ & 227.022 & 0.023 & 0.004 & 0.001 & 0.010 & 0.002 & 0.000 \\
\hline \multirow[t]{3}{*}{${ }^{31} \mathrm{P}$} & $\mathrm{PH}_{3}$ & 598.967 & 0.081 & 0.014 & 0.003 & 0.013 & 0.002 & 0.001 \\
\hline & $\mathrm{PF}_{3}$ & 189.238 & 0.007 & 0.001 & 0.000 & 0.004 & 0.000 & 0.000 \\
\hline & $\mathrm{PN}$ & -44.886 & 0.023 & 0.004 & 0.001 & 0.052 & 0.010 & 0.002 \\
\hline \multirow{3}{*}{\multicolumn{2}{|c|}{$\begin{array}{l}\text { MAE/MARE }\left({ }^{1} \mathrm{H}\right) \\
\text { MAE/MARE }\left({ }^{13} \mathrm{C}\right) \\
\text { MAE/MARE (other) }\end{array}$}} & & 0.001 & 0.000 & 0.000 & 0.002 & 0.001 & 0.000 \\
\hline & & & 0.012 & 0.003 & 0.001 & 0.025 & 0.006 & 0.001 \\
\hline & & & 0.028 & 0.006 & 0.001 & 0.032 & 0.008 & 0.002 \\
\hline
\end{tabular}


Table S15: Absolute $\Delta \sigma_{a b s}$ (in ppm) and relative deviations $\Delta \sigma_{r e l}$ (in \%) due to combined screening thresholds for the Lh07t-SVWN functional

\begin{tabular}{|c|c|c|c|c|c|c|c|c|}
\hline com & $\begin{array}{l}\text { ned S-, P-, F-, } \\
\text { molecule }\end{array}$ & $\begin{array}{l}\text { PL- and FL-ju } \\
\text { no screening }\end{array}$ & $\begin{array}{l}\text { ctions } \\
\text { low }^{a}\end{array}$ & $\Delta \sigma_{a b s}(\mathrm{ppm})$ & $\Delta \sigma_{r e l}(\%)$ & $\operatorname{high}^{b}$ & $\Delta \sigma_{a b s}(\mathrm{ppm})$ & $\Delta \sigma_{r e l}(\%)$ \\
\hline \multirow[t]{8}{*}{${ }^{1} \mathrm{H}$} & furan $(\mathrm{C} 2 / 5)$ & 23.681 & 23.681 & 0.001 & 0.003 & 23.680 & 0.001 & 0.004 \\
\hline & furan $(\mathrm{C} 3 / 4)$ & 24.626 & 24.634 & 0.008 & 0.033 & 24.626 & 0.000 & 0.001 \\
\hline & $\left(\mathrm{CH}_{3}\right)_{2} \mathrm{CO}$ & 29.283 & 29.285 & 0.003 & 0.009 & 29.283 & 0.000 & 0.001 \\
\hline & $\mathrm{CH}_{4}$ & 31.307 & 31.309 & 0.001 & 0.004 & 31.308 & 0.000 & 0.000 \\
\hline & $\mathrm{NH}_{3}$ & 31.326 & 31.325 & 0.001 & 0.005 & 31.327 & 0.000 & 0.001 \\
\hline & $\mathrm{H}_{2} \mathrm{O}$ & 30.474 & 30.479 & 0.006 & 0.019 & 30.475 & 0.001 & 0.003 \\
\hline & $\mathrm{HF}$ & 28.552 & 28.552 & 0.000 & 0.001 & 28.555 & 0.002 & 0.008 \\
\hline & $\mathrm{PH}_{3}$ & 29.251 & 29.257 & 0.006 & 0.020 & 29.252 & 0.000 & 0.001 \\
\hline \multirow[t]{7}{*}{${ }^{13} \mathrm{C}$} & furan $(\mathrm{C} 2 / 5)$ & 38.015 & 37.956 & 0.059 & 0.156 & 38.012 & 0.003 & 0.009 \\
\hline & furan $(\mathrm{C} 3 / 4)$ & 72.317 & 72.333 & 0.016 & 0.023 & 72.317 & 0.001 & 0.001 \\
\hline & $\left(\mathrm{CH}_{3}\right)_{2} \underline{\mathrm{CO}}$ & -30.312 & -30.329 & 0.017 & 0.057 & -30.306 & 0.006 & 0.019 \\
\hline & $\left(\underline{\mathrm{CH}}_{3}\right)_{2} \overline{\mathrm{CO}}$ & 159.382 & 159.414 & 0.033 & 0.020 & 159.384 & 0.002 & 0.001 \\
\hline & $\mathrm{CH}_{4}$ & 199.852 & 199.858 & 0.006 & 0.003 & 199.854 & 0.002 & 0.001 \\
\hline & $\mathrm{CF}_{4}$ & 53.404 & 53.243 & 0.161 & 0.301 & 53.403 & 0.001 & 0.002 \\
\hline & $\mathrm{CO}$ & -13.202 & -13.182 & 0.020 & 0.149 & -13.199 & 0.003 & 0.021 \\
\hline \multirow[t]{5}{*}{${ }^{15} \mathrm{~N}$} & $\mathrm{~N}_{2}$ & -81.593 & -81.582 & 0.011 & 0.013 & -81.554 & 0.039 & 0.048 \\
\hline & $\mathrm{NH}_{3}$ & 275.845 & 275.808 & 0.037 & 0.013 & 275.857 & 0.012 & 0.004 \\
\hline & NNO & -1.517 & -1.513 & 0.004 & 0.234 & -1.510 & 0.007 & 0.487 \\
\hline & $\underline{\mathrm{NNO}}$ & 94.444 & 94.515 & 0.071 & 0.075 & 94.457 & 0.013 & 0.014 \\
\hline & $\overline{\mathrm{PN}}$ & -401.714 & -401.846 & 0.132 & 0.033 & -401.722 & 0.008 & 0.002 \\
\hline \multirow[t]{6}{*}{${ }^{17} \mathrm{O}$} & furan & 37.547 & 37.641 & 0.094 & 0.251 & 37.572 & 0.025 & 0.067 \\
\hline & $\left(\mathrm{CH}_{3}\right)_{2} \mathrm{CO}$ & -316.519 & -316.658 & 0.139 & 0.044 & -316.558 & 0.038 & 0.012 \\
\hline & $\mathrm{H}_{2} \mathrm{O}$ & 349.495 & 349.735 & 0.241 & 0.069 & 349.517 & 0.022 & 0.006 \\
\hline & $\mathrm{CO}$ & -64.958 & -65.228 & 0.271 & 0.416 & -64.976 & 0.018 & 0.028 \\
\hline & $\mathrm{OF}_{2}$ & -538.324 & -538.189 & 0.135 & 0.025 & -538.295 & 0.028 & 0.005 \\
\hline & $\mathrm{NNO}$ & 191.339 & 191.031 & 0.308 & 0.161 & 191.335 & 0.004 & 0.002 \\
\hline \multirow[t]{5}{*}{${ }^{19} \mathrm{~F}$} & $\mathrm{HF}$ & 437.037 & 437.690 & 0.653 & 0.149 & 437.081 & 0.044 & 0.010 \\
\hline & $\mathrm{F}_{2}$ & -215.668 & -215.659 & 0.009 & 0.004 & -215.653 & 0.015 & 0.007 \\
\hline & $\mathrm{CF}_{4}$ & 262.882 & 262.882 & 0.000 & 0.000 & 262.889 & 0.008 & 0.003 \\
\hline & $\mathrm{OF}_{2}$ & -34.200 & -34.309 & 0.108 & 0.317 & -34.187 & 0.013 & 0.038 \\
\hline & $\mathrm{PF}_{3}$ & 227.022 & 226.943 & 0.079 & 0.035 & 227.024 & 0.002 & 0.001 \\
\hline \multirow[t]{3}{*}{${ }^{31} \mathrm{P}$} & $\mathrm{PH}_{3}$ & 598.967 & 598.806 & 0.162 & 0.027 & 598.971 & 0.003 & 0.001 \\
\hline & $\mathrm{PF}_{3}$ & 189.238 & 189.317 & 0.079 & 0.042 & 189.220 & 0.018 & 0.009 \\
\hline & $\mathrm{PN}$ & -44.886 & -44.307 & 0.579 & 1.289 & -44.861 & 0.024 & 0.054 \\
\hline \multirow{3}{*}{\multicolumn{3}{|c|}{$\begin{array}{l}\text { MAE/MARE }\left({ }^{1} \mathrm{H}\right) \\
\text { MAE/MARE }\left({ }^{13} \mathrm{C}\right) \\
\text { MAE/MARE (other) }\end{array}$}} & & 0.003 & 0.012 & & 0.001 & 0.002 \\
\hline & & & & 0.045 & 0.101 & & 0.003 & 0.008 \\
\hline & & & & 0.164 & 0.168 & & 0.018 & 0.042 \\
\hline
\end{tabular}

${ }^{a}$ Low: S/P-junctions $10^{-3}$, F-junctions $10^{-7}$, PL-junctions $10^{-5}$, FL-junctions $10^{-9}$.

${ }^{b}$ High: S/P-junctions $10^{-4}$, F-junctions $10^{-8}$, PL-junctions $10^{-6}$, FL-junctions $10^{-10}$. 
Table S16: Top: numbering of nuclei in the $\mathrm{C}_{10} \mathrm{H}_{12}$ molecule. Bottom: full results of $\mathrm{C}_{10} \mathrm{H}_{12}$ shielding calculation employing different screening settings (Lh07t-SVWN/def2-TZVP/MARI-J level), with and without symmetry

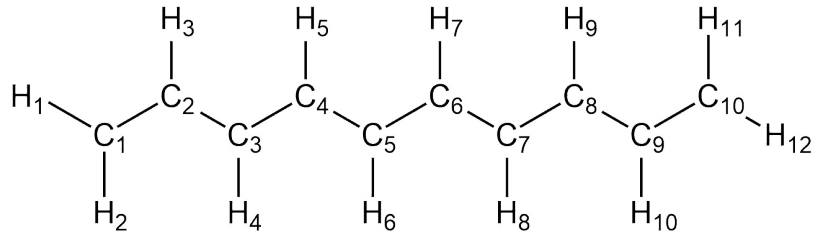

\begin{tabular}{|c|c|c|c|c|c|c|}
\hline & \multirow[b]{2}{*}{ nucleus } & \multirow[b]{2}{*}{ no screening } & \multicolumn{2}{|c|}{ symmetry $\mathrm{C}_{1}$} & \multicolumn{2}{|c|}{ symmetry $\mathrm{C}_{s}$} \\
\hline & & & low $^{a}$ & $\operatorname{high}^{b}$ & $\operatorname{low}^{a}$ & $\operatorname{high}^{b}$ \\
\hline \multirow[t]{12}{*}{${ }^{1} \mathrm{H}$} & $\mathrm{H}_{1}$ & 26.267 & 26.271 & 26.267 & 26.257 & 26.262 \\
\hline & $\mathrm{H}_{2}$ & 25.984 & 26.000 & 25.983 & 25.996 & 25.992 \\
\hline & $\mathrm{H}_{3}$ & 24.661 & 24.614 & 24.654 & 24.662 & 24.661 \\
\hline & $\mathrm{H}_{4}$ & 24.819 & 24.850 & 24.818 & 24.803 & 24.819 \\
\hline & $\mathrm{H}_{5}$ & 24.852 & 24.810 & 24.847 & 24.866 & 24.851 \\
\hline & $\mathrm{H}_{6}$ & 24.849 & 24.847 & 24.849 & 24.842 & 24.848 \\
\hline & $\mathrm{H}_{7}$ & 24.849 & 24.844 & 24.848 & 24.845 & 24.848 \\
\hline & $\mathrm{H}_{8}$ & 24.852 & 24.867 & 24.861 & 24.867 & 24.851 \\
\hline & $\mathrm{H}_{9}$ & 24.819 & 24.778 & 24.806 & 24.812 & 24.819 \\
\hline & $\mathrm{H}_{10}$ & 24.661 & 24.693 & 24.674 & 24.678 & 24.661 \\
\hline & $\mathrm{H}_{11}$ & 25.984 & 25.997 & 25.993 & 25.993 & 25.992 \\
\hline & $\mathrm{H}_{12}$ & 26.267 & 26.249 & 26.263 & 26.255 & 26.262 \\
\hline \multirow[t]{10}{*}{${ }^{13} \mathrm{C}$} & $\mathrm{C}_{1}$ & 69.889 & 69.984 & 69.899 & 69.816 & 69.865 \\
\hline & $\mathrm{C}_{2}$ & 42.866 & 42.966 & 42.888 & 42.836 & 42.848 \\
\hline & $\mathrm{C}_{3}$ & 48.443 & 48.437 & 48.449 & 48.399 & 48.447 \\
\hline & $\mathrm{C}_{4}$ & 48.386 & 48.404 & 48.408 & 48.375 & 48.394 \\
\hline & $\mathrm{C}_{5}$ & 48.453 & 48.434 & 48.455 & 48.468 & 48.462 \\
\hline & $\mathrm{C}_{6}$ & 48.453 & 48.415 & 48.474 & 48.467 & 48.462 \\
\hline & $\mathrm{C}_{7}$ & 48.386 & 48.300 & 48.370 & 48.361 & 48.394 \\
\hline & $\mathrm{C}_{8}$ & 48.443 & 48.324 & 48.463 & 48.403 & 48.447 \\
\hline & $\mathrm{C}_{9}$ & 42.865 & 42.779 & 42.801 & 42.829 & 42.849 \\
\hline & $\mathrm{C}_{10}$ & 69.889 & 69.702 & 69.837 & 69.814 & 69.865 \\
\hline \multirow{2}{*}{\multicolumn{2}{|c|}{$\begin{array}{l}\operatorname{MAE}\left({ }^{1} \mathrm{H}\right) \\
\operatorname{MAE}\left({ }^{13} \mathrm{C}\right)\end{array}$}} & & 0.022 & 0.005 & 0.011 & 0.003 \\
\hline & & & 0.075 & 0.024 & 0.036 & 0.013 \\
\hline
\end{tabular}

${ }^{a}$ Low: S/P- $10^{-3}$, F- $10^{-7}$ PL- $10^{-5}$, FL-junctions $10^{-9}$.

${ }^{b}$ High: S/P- $10^{-4}$, F- $10^{-8}$ PL- $10^{-6}$, FL-junctions $10^{-10}$. 
Table S17: Isotropic shielding constants calculated at Lh07t-SVWN/pcSseg-4 level compared to CCSD(T) reference data, using different grid and pre-screening settings.

\begin{tabular}{|c|c|c|c|c|c|}
\hline & molecule & full $^{\mathrm{a}}$ & low ${ }^{b}$ & high $^{\mathrm{b}}$ & ref. \\
\hline \multirow[t]{8}{*}{${ }^{1} \mathrm{H}$} & furan $(\mathrm{C} 2 / 5)$ & 23.68 & 23.69 & 23.68 & 24.03 \\
\hline & furan $(\mathrm{C} 3 / 4)$ & 24.63 & 24.63 & 24.63 & 25.02 \\
\hline & $\left(\mathrm{CH}_{3}\right)_{2} \mathrm{CO}$ & 29.28 & 29.28 & 29.28 & 29.53 \\
\hline & $\mathrm{CH}_{4}$ & 31.31 & 31.31 & 31.31 & 31.39 \\
\hline & $\mathrm{NH}_{3}$ & 31.33 & 31.33 & 31.33 & 31.44 \\
\hline & $\mathrm{H}_{2} \mathrm{O}$ & 30.47 & 30.48 & 30.47 & 30.65 \\
\hline & $\mathrm{HF}$ & 28.55 & 28.56 & 28.55 & 28.82 \\
\hline & $\mathrm{PH}_{3}$ & 29.25 & 29.26 & 29.25 & 29.46 \\
\hline \multirow[t]{7}{*}{${ }^{13} \mathrm{C}$} & furan $(\mathrm{C} 2 / 5)$ & 38.02 & 37.98 & 38.01 & 47.36 \\
\hline & furan (C3/4) & 72.32 & 72.41 & 72.40 & 81.67 \\
\hline & $\left(\mathrm{CH}_{3}\right)_{2} \underline{\mathrm{CO}}$ & -30.31 & -30.38 & -30.36 & -10.84 \\
\hline & $\left(\underline{\mathrm{CH}}_{3}\right)_{2} \mathrm{CO}$ & 159.38 & 159.43 & 159.43 & 162.88 \\
\hline & $\overline{\mathrm{CH}}_{4}$ & 199.85 & 199.83 & 199.82 & 199.39 \\
\hline & $\mathrm{CF}_{4}$ & 53.40 & 53.31 & 53.43 & 65.96 \\
\hline & $\mathrm{CO}$ & -13.20 & -13.13 & -13.17 & 2.56 \\
\hline \multirow[t]{5}{*}{${ }^{15} \mathrm{~N}$} & $\mathrm{~N}_{2}$ & -81.59 & -81.51 & -81.50 & -61.16 \\
\hline & $\mathrm{NH}_{3}$ & 275.84 & 275.77 & 275.77 & 270.40 \\
\hline & NNO & -1.52 & -1.48 & -1.49 & 11.74 \\
\hline & $\underline{\mathrm{NNO}}$ & 94.44 & 94.49 & 94.49 & 106.22 \\
\hline & $\overline{\mathrm{PN}}$ & -401.71 & -401.79 & -401.75 & -344.71 \\
\hline \multirow[t]{6}{*}{${ }^{17} \mathrm{O}$} & furan & 37.55 & 37.51 & 37.45 & 64.82 \\
\hline & $\left(\mathrm{CH}_{3}\right)_{2} \mathrm{CO}$ & -316.52 & -316.76 & -316.79 & -297.91 \\
\hline & $\mathrm{H}_{2} \mathrm{O}$ & 349.49 & 349.50 & 349.47 & 337.63 \\
\hline & $\mathrm{CO}$ & -64.96 & -64.92 & -64.97 & -55.42 \\
\hline & $\mathrm{OF}_{2}$ & -538.32 & -538.45 & -538.43 & -446.32 \\
\hline & $\mathrm{NNO}$ & 191.34 & 191.30 & 191.34 & 198.77 \\
\hline \multirow[t]{5}{*}{${ }^{19} \mathrm{~F}$} & $\mathrm{HF}$ & 437.04 & 437.07 & 437.05 & 419.91 \\
\hline & $\mathrm{F}_{2}$ & -215.67 & -215.49 & -215.49 & -192.76 \\
\hline & $\mathrm{CF}_{4}$ & 262.88 & 263.09 & 263.05 & 267.58 \\
\hline & $\mathrm{OF}_{2}$ & -34.20 & -34.12 & -34.06 & -24.28 \\
\hline & $\mathrm{PF}_{3}$ & 227.02 & 227.03 & 227.04 & 231.81 \\
\hline \multirow[t]{3}{*}{${ }^{31} \mathrm{P}$} & $\mathrm{PH}_{3}$ & 598.97 & 598.58 & 598.58 & 604.51 \\
\hline & $\mathrm{PF}_{3}$ & 189.24 & 189.19 & 189.12 & 224.80 \\
\hline & $\mathrm{PN}$ & -44.89 & -44.92 & -44.96 & 51.61 \\
\hline \multicolumn{2}{|c|}{$\operatorname{MSE}\left({ }^{1} \mathrm{H}\right)$} & -0.23 & -0.22 & -0.23 & \\
\hline \multicolumn{2}{|c|}{$\mathrm{MSE}\left({ }^{13} \mathrm{C}\right)$} & -9.93 & -9.94 & -9.92 & \\
\hline \multicolumn{2}{|c|}{ MSE (other) } & -21.20 & -21.22 & -21.23 & \\
\hline \multicolumn{2}{|c|}{$\operatorname{MAE}\left({ }^{1} \mathrm{H}\right)$} & 0.23 & 0.22 & 0.23 & \\
\hline \multicolumn{2}{|c|}{ MAE $\left({ }^{13} \mathrm{C}\right)$} & 10.06 & 10.06 & 10.04 & \\
\hline \multicolumn{2}{|c|}{ MAE (other) } & 24.83 & 24.84 & 24.84 & \\
\hline \multicolumn{2}{|c|}{$\mathrm{SD}\left({ }^{1} \mathrm{H}\right)$} & 0.10 & 0.10 & 0.10 & \\
\hline \multicolumn{2}{|c|}{$\mathrm{SD}\left({ }^{13} \mathrm{C}\right)$} & 6.35 & 6.36 & 6.35 & \\
\hline \multicolumn{2}{|c|}{ SD (other) } & 29.81 & 29.83 & 29.83 & \\
\hline
\end{tabular}

${ }^{a}$ Grid 5 without any pre-screening.

${ }^{b}$ Low: grid 1 (SCF \& shieldings)/grid 0 (CPKS-loop), S/P-junctions $10^{-3}$, F-junctions $10^{-7}$, PL-junctions $10^{-5}$, FL-junctions $10^{-9}$.

${ }^{c}$ High: grid 1 (SCF \& shieldings)/grid 0 (CPKS-loop), S/P-junctions $10^{-4}$, F-junctions $10^{-8}$, PL-junctions $10^{-6}$, FL-junctions $10^{-10}$. 
Table S18: Isotropic shielding constants calculated for two LHs and the corresponding GHs obtained by choosing the maximum EX admixture of the LH. Statistical data from comparison to the CCSD(T) reference values provided in the last column.

\begin{tabular}{|c|c|c|c|c|c|c|}
\hline & molecule & Lh07t-SVWN (07t) & GH-07t-max & Lh12ct-SsirPW92 (12sir) & GH-12sir-max & ref. \\
\hline \multirow[t]{8}{*}{${ }^{1} \mathrm{H}$} & furan $(\mathrm{C} 2 / 5)$ & 23.68 & 23.82 & 23.52 & 23.70 & 24.03 \\
\hline & furan $(\mathrm{C} 3 / 4)$ & 24.63 & 24.76 & 24.45 & 24.62 & 25.02 \\
\hline & $\left(\mathrm{CH}_{3}\right)_{2} \mathrm{CO}$ & 29.28 & 29.43 & 29.19 & 29.37 & 29.53 \\
\hline & $\mathrm{CH}_{4}$ & 31.31 & 31.41 & 31.19 & 31.32 & 31.39 \\
\hline & $\mathrm{NH}_{3}$ & 31.33 & 31.44 & 31.17 & 31.32 & 31.44 \\
\hline & $\mathrm{H}_{2} \mathrm{O}$ & 30.47 & 30.54 & 30.28 & 30.36 & 30.65 \\
\hline & $\mathrm{HF}$ & 28.55 & 28.50 & 28.28 & 28.21 & 28.82 \\
\hline & $\mathrm{PH}_{3}$ & 29.25 & 29.39 & 29.12 & 29.31 & 29.46 \\
\hline \multirow[t]{7}{*}{${ }^{13} \mathrm{C}$} & furan $(\mathrm{C} 2 / 5)$ & 38.02 & 28.23 & 42.06 & 29.15 & 47.36 \\
\hline & furan (C3/4) & 72.32 & 64.06 & 76.39 & 65.49 & 81.67 \\
\hline & $\left(\mathrm{CH}_{3}\right)_{2} \underline{\mathrm{CO}}$ & -30.31 & -37.44 & -25.88 & -35.53 & -10.84 \\
\hline & $\left(\mathrm{CH}_{3}\right)_{2} \overline{\mathrm{CO}}$ & 159.38 & 154.52 & 163.67 & 157.26 & 162.88 \\
\hline & $\overline{\mathrm{CH}}_{4}$ & 199.85 & 193.34 & 203.92 & 195.33 & 199.39 \\
\hline & $\mathrm{CF}_{4}$ & 53.40 & 56.56 & 58.82 & 63.07 & 65.96 \\
\hline & $\mathrm{CO}$ & -13.20 & -30.20 & -10.87 & -33.97 & 2.56 \\
\hline \multirow[t]{5}{*}{${ }^{15} \mathrm{~N}$} & $\mathrm{~N}_{2}$ & -81.59 & -110.04 & -78.74 & -117.43 & -61.16 \\
\hline & $\mathrm{NH}_{3}$ & 275.84 & 263.15 & 280.43 & 263.58 & 270.4 \\
\hline & NNO & -1.52 & -22.70 & 0.70 & -27.60 & 11.74 \\
\hline & $\underline{\mathrm{NNO}}$ & 94.44 & 69.72 & 97.76 & 64.57 & 106.22 \\
\hline & $\overline{\mathrm{PN}}$ & -401.71 & -478.56 & -395.99 & -501.26 & -344.71 \\
\hline \multirow[t]{6}{*}{${ }^{17} \mathrm{O}$} & furan & 37.55 & 25.73 & 44.76 & 30.11 & 64.82 \\
\hline & $\left(\mathrm{CH}_{3}\right)_{2} \mathrm{CO}$ & -316.52 & -366.35 & -306.17 & -371.98 & -297.91 \\
\hline & $\mathrm{H}_{2} \mathrm{O}$ & 349.49 & 330.06 & 354.87 & 328.98 & 337.63 \\
\hline & $\mathrm{CO}$ & -64.96 & -96.61 & -60.21 & -103.19 & -55.42 \\
\hline & $\mathrm{OF}_{2}$ & -538.32 & -544.92 & -511.64 & -526.48 & -446.32 \\
\hline & $\mathrm{NNO}$ & 191.34 & 170.87 & 196.51 & 168.90 & 198.77 \\
\hline \multirow[t]{5}{*}{${ }^{19} \mathrm{~F}$} & $\mathrm{HF}$ & 437.04 & 415.11 & 442.80 & 413.63 & 419.91 \\
\hline & $\mathrm{F}_{2}$ & -215.67 & -238.09 & -198.22 & -228.16 & -192.76 \\
\hline & $\mathrm{CF}_{4}$ & 262.88 & 255.19 & 271.40 & 260.64 & 267.58 \\
\hline & $\mathrm{OF}_{2}$ & -34.20 & -39.76 & -16.18 & -23.98 & -24.28 \\
\hline & $\mathrm{PF}_{3}$ & 227.02 & 218.40 & 238.06 & 225.90 & 231.81 \\
\hline \multirow[t]{3}{*}{${ }^{31} \mathrm{P}$} & $\mathrm{PH}_{3}$ & 598.97 & 576.87 & 615.34 & 585.85 & 604.51 \\
\hline & $\mathrm{PF}_{3}$ & 189.24 & 189.80 & 204.92 & 205.59 & 224.8 \\
\hline & $\mathrm{PN}$ & -44.89 & -113.02 & -40.28 & -133.04 & 51.61 \\
\hline \multicolumn{2}{|c|}{$\operatorname{MSE}\left({ }^{1} \mathrm{H}\right)$} & -0.23 & -0.13 & -0.39 & -0.26 & \\
\hline \multicolumn{2}{|c|}{$\operatorname{MSE}\left({ }^{13} \mathrm{C}\right)$} & -9.93 & -17.13 & -5.84 & -15.46 & \\
\hline \multicolumn{2}{|c|}{ MSE (other) } & -21.20 & -45.39 & -11.95 & -44.87 & \\
\hline \multicolumn{2}{|c|}{ MAE $\left({ }^{1} \mathrm{H}\right)$} & 0.23 & 0.14 & 0.39 & 0.26 & \\
\hline \multicolumn{2}{|c|}{ MAE $\left({ }^{13} \mathrm{C}\right)$} & 10.06 & 17.13 & 7.36 & 15.46 & \\
\hline \multicolumn{2}{|c|}{ MAE (other) } & 24.83 & 45.39 & 20.29 & 44.91 & \\
\hline \multicolumn{2}{|c|}{$\mathrm{SD}\left({ }^{1} \mathrm{H}\right)$} & 0.10 & 0.11 & 0.13 & 0.17 & \\
\hline \multicolumn{2}{|c|}{$\mathrm{SD}\left({ }^{13} \mathrm{C}\right)$} & 6.35 & 9.24 & 6.50 & 11.48 & \\
\hline \multicolumn{2}{|c|}{ SD (other) } & 29.81 & 42.27 & 28.29 & 48.83 & \\
\hline
\end{tabular}


Table S19: Results of shielding calculations for TPSS and M06L ${ }^{2}$ (in ppm) using different programs

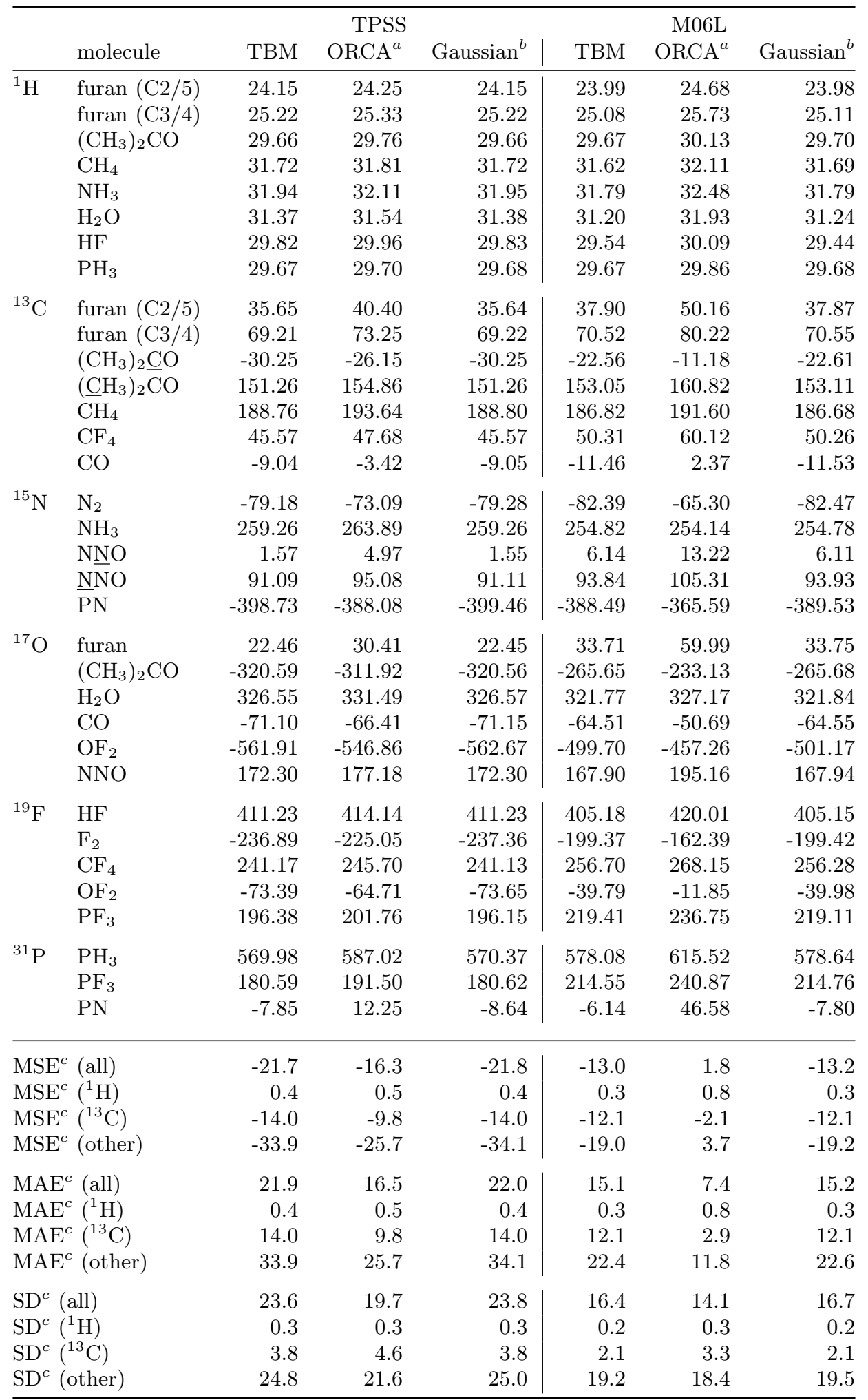

${ }^{a}$ Data taken from Supporting Information of ref. 3.

${ }^{b}$ Gaussian 09.D01 ${ }^{4}$ calculation.

${ }^{c}$ Statistics relative to the $\operatorname{CCSD}(\mathrm{T})$ reference data of ref. 3 . 

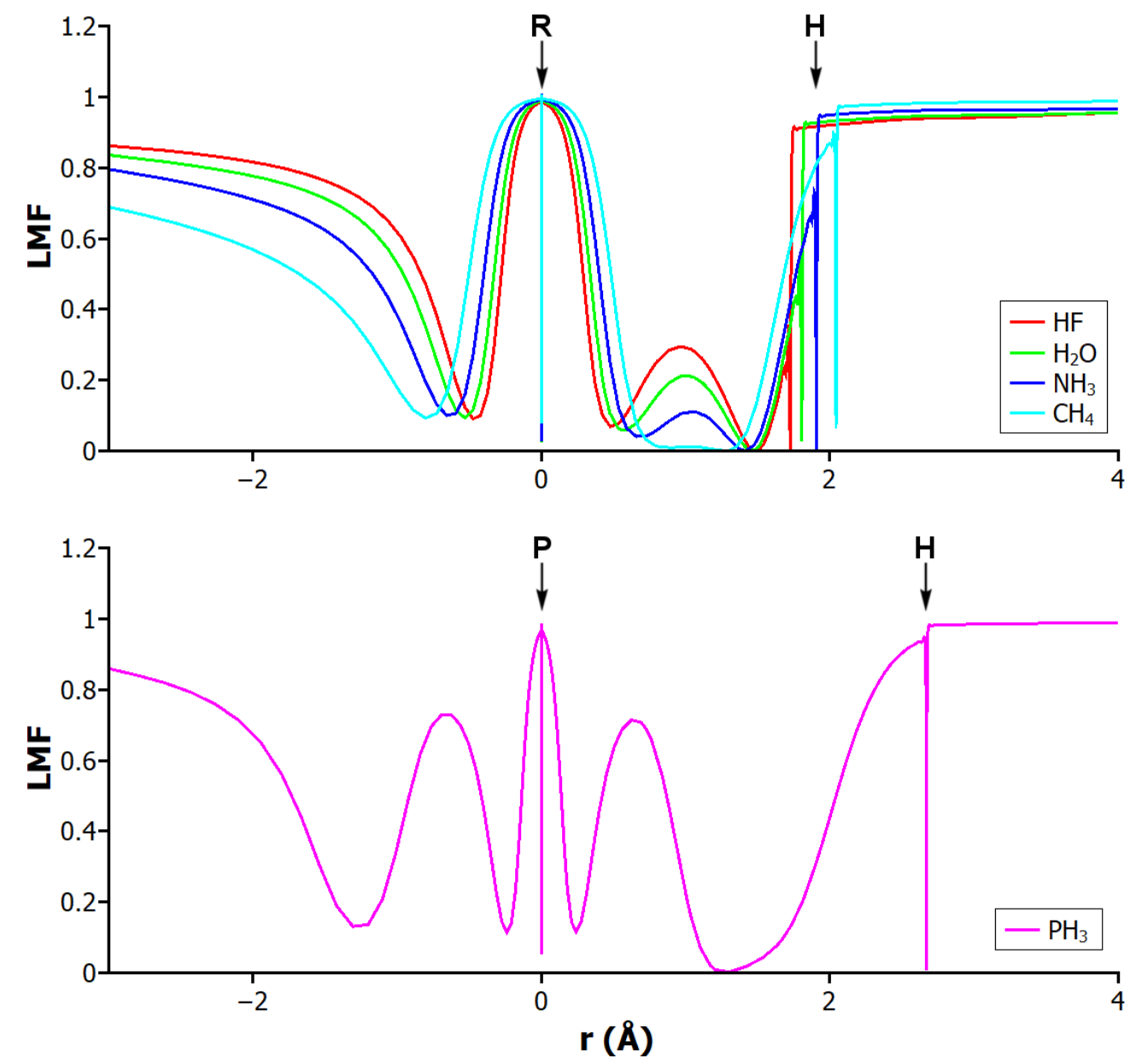

Figure S3: Plots of the (unscaled) t-LMF along different E-H bonds. Top: Combined $\mathrm{CH}_{4}, \mathrm{NH}_{3}, \mathrm{H}_{2} \mathrm{O}$, and $\mathrm{HF}$. Bottom: $\mathrm{PH}_{3}$. The E-atom is placed at the origin, while the hydrogen atom is generally on the right side of the plot. 


\section{References}

(1) Helgaker, T.; Jørgensen, P.; Olsen, J. Molecular Electronic-Structure Theory, 1st ed.; John Wiley \& Sons Ltd: Chichester, 2000.

(2) Zhao, Y.; Truhlar, D. G. A new local density functional for main-group thermochemistry, transition metal bonding, thermochemical kinetics, and noncovalent interactions. J. Chem. Phys. 2006, 125, 194101.

(3) Stoychev, G. L.; Auer, A. A.; R., I.; Neese, F. Self-Consistent Field Calculation of Nuclear Magnetic Resonance Chemical Shielding Constants Using Gauge-Including Atomic Orbitals and Approximate Two-Electron Integrals. J. Chem. Theory Comput. 2018, 14, 619-637.

(4) Gaussian 09, Revision D.01, M. J. Frisch, G. W. Trucks, H. B. Schlegel, G. E. Scuseria, M. A. Robb, J. R. Cheeseman, G. Scalmani, V. Barone, B. Mennucci, G. A. Petersson, H. Nakatsuji, M. Caricato, X. Li, H. P. Hratchian, A. F. Izmaylov, J. Bloino, G. Zheng, J. L. Sonnenberg, M. Hada, M. Ehara, K. Toyota, R. Fukuda, J. Hasegawa, M. Ishida, T. Nakajima, Y. Honda, O. Kitao, H. Nakai, T. Vreven, J. A. Montgomery, Jr., J. E. Peralta, F. Ogliaro, M. Bearpark, J. J. Heyd, E. Brothers, K. N. Kudin, V. N. Staroverov, T. Keith, R. Kobayashi, J. Normand, K. Raghavachari, A. Rendell, J. C. Burant, S. S. Iyengar, J. Tomasi, M. Cossi, N. Rega, J. M. Millam, M. Klene, J. E. Knox, J. B. Cross, V. Bakken, C. Adamo, J. Jaramillo, R. Gomperts, R. E. Stratmann, O. Yazyev, A. J. Austin, R. Cammi, C. Pomelli, J. W. Ochterski, R. L. Martin, K. Morokuma, V. G.

Zakrzewski, G. A. Voth, P. Salvador, J. J. Dannenberg, S. Dapprich, A. D. Daniels, O. Farkas, J. B. Foresman, J. V. Ortiz, J. Cioslowski, and D. J. Fox, Gaussian, Inc., Wallingford CT, 2013. 